\title{
Categorias COM MORFismos Verdade E PRototopos: UmA SEMÂNTICA PARA LINGUAGENS PROPOSICIONAIS ${ }^{1}$
}

\author{
Edelcio G. de Souza ${ }^{2}$
}

\begin{abstract}
O objetivo do presente trabalho é desenvolver algumas propriedades de categorias que são mais gerais que categorias que são topos. Apresentamos, assim, os conceitos de categorias com morfismos verdade (CTM) e prototopos, mostrando como se podem definir os morfismos verdade nessas categorias de modo a prover uma semântica para linguagens proposicionais usuais. Mostramos, também, que em categorias com morfismos verdade vale um teorema análogo ao teorema fundamental da teoria de topos.
\end{abstract}

\section{Introdução}

A fim de acompanhar o presente trabalho, assumimos que o leitor esteja familiarizado com o conceitos básicos de teoria de categorias, incluindo a noção de limite e colimite, classificadores de subobjetos e exponenciação ${ }^{3}$. Esperamos, assim, que o leitor conheça as propriedades fundamentais de categorias que são topos ${ }^{4}$. O objetivo é introduzir um tipo mais geral de categoria, que denominaremos prototopos, que constitua um ambiente em que se possa construir semântica para linguagens proposicionais ${ }^{5}$. Precisamos, então, de um tipo de categoria em que os valores de verdade podem ser definidos junto com os morfismos verdade que correspondem às funções de verdade usuais como negação, conjunção, implicação e disjunção. Vamos mostrar que para estas construções não precisamos que a categoria seja cartesiana fechada, em particular que tenha exponenciação, mas apenas que ela satisfaça uma propriedade dessas categorias, a saber, o que denominamos de 0-Axioma: se um morfismo possui como codomínio um objeto inicial, então ele é um isomorfismo.

Segue-se do 0-Axioma, que se o domínio de um morfismo é um objeto inicial, então ele deve ser um monomorfismo. Isso implica que o morfismo

$$
0 \rightarrow 1
$$

deve ser um monomorfismo. Assim, assumindo que a categoria possui classificador de subobjetos $T: 1 \rightarrow \Omega$, o morfismo acima possui um caráter dado por $\perp: 1 \rightarrow \Omega$, que é identificado como o valor de verdade falso.

Se a categoria é não degenerada, temos assim dois valores de verdade diferentes, $\top, \perp$ : $1 \rightarrow \Omega$, que correspondem ao verdadeiro e ao falso.

\footnotetext{
${ }^{1}$ Esse trabalho foi desenvolvido durante um estágio de pesquisa na Universidade de Miami. Agradeço ao Prof. Dr. Otávio Bueno por sua hospitalidade e à Fundação de Amparo à Pesquisa do Estado de São Paulo pela concessão da bolsa de pesquisa no exterior, processo número FAPESP 2018/10326-4.

2 Departamento de Filosofia, FFLCH, USP. Email: edelcio.souza@usp.br.

${ }^{3}$ Vamos utilizar a notação de Goldblatt [6].

${ }^{4} \mathrm{O}$ leitor pode consultar além de Goldblatt [6], os seguintes textos fundamentais: Awodey [1], Bell [2], Herrlich \& Strecker [7] e MacLane [9]. Para a teoria de topos ver: MacLane \& Moerdijik [10] e MacLarty [11].

${ }^{5}$ Uma versão preliminar e resumida do presente trabalho pode ser vista em de Souza [4].

DOI: 10.21452/LnA_serie_n_v01_book_seminario-logica-no-aviao-2013-2018_edelcio-souza_p.73-102
} 
Com o falso, é fácil construir a função de verdade que corresponde à negação. Como $\perp: 1 \rightarrow \Omega$ é um monomorfismo, seu caráter é dado por $\chi_{\perp}=f_{\neg}: \Omega \rightarrow \Omega$ que é o morfismo verdade que corresponde à negação. Para ver isso, é suficiente mostrar que $f_{\neg} \circ \top=\perp$ e $f_{\neg} \circ \perp=\top$ e temos o comportamento usual da função de verdade que corresponde à negação com respeito aos valores de verdade verdadeiro e falso.

Vamos mostrar que podemos fazer as mesmas construções para os morfismos que correspondem às outras funções usuais de verdade, e apresentar como se comportam com respeito aos valores de verdade. Para isto definiremos, por meio de axiomas, dois tipos de categorias em que todas essas construções podem ser realizadas.

\section{Categorias com morfismos verdade}

Dizemos que uma categoria $\mathcal{E}$ é uma categoria com morfismos verdade (CTM) se e somente se ela satisfaz os seguintes axiomas:

$(\mathrm{CTM} 1) \mathcal{E}$ é finitamente completa;

$(\mathrm{CTM} 2) \mathcal{E}$ é finitamente cocompleta;

(CTM3) $\mathcal{E}$ possui classificador de subobjetos;

$(\mathrm{CTM} 4) \mathcal{E}$ satisfaz a seguinte propriedade: (0-Axioma) Se existe um $\mathcal{E}$-morfismo $f$ : $a \rightarrow 0$ (0 é inicial em $\mathcal{E}$ ), $f$ é um isomorfismo. E, então, temos que $a$ e 0 são isomorfos, e $a$ é também inicial em $\mathcal{E}$.

Uma categoria $\mathcal{E}$ é dita finitamente bicompleta (completa e cocompleta) quando todo diagrama finito em $\mathcal{E}$ possui limite e colimite. Nesse caso, $\mathcal{E}$ possui objetos terminais e iniciais e, em $\mathcal{E}$, pode-se fazer uma série de construções úteis: produtos e coprodutos de objetos e morfismos, equalizadores e coequalizadores, pullbacks e pushouts, etc.

Considere um objeto $d$ em uma categoria $\mathcal{E}$. Um subobjeto de $d$ é uma certa classe de equivalência de monomorfismos com codomínio $d$. Sejam os $\mathcal{E}$-monomorfismos $f: a \longmapsto d$ e $g: b \longmapsto d$. Dizemos que $f$ é equivalente a $g$, em símbolos $f \equiv g$ se existe um isomorfismo $h: a \rightarrow b$ tal que o diagrama

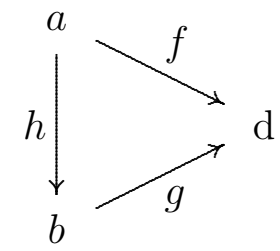

comuta, isto é, $f=g \circ h$.

É fácil ver que $\equiv$ é uma relação de equivalência e, se $M o n(d)$ é a classe dos monomorfismos com domínio $d$, então um subobjeto de $d$ é qualquer elemento do conjunto quociente:

$$
\operatorname{Mon}(d) / \equiv:=\left\{[f]_{\equiv}: f \in \operatorname{Mon}(d)\right\} .
$$

Se $\mathcal{E}$ é uma categoria com objeto terminal 1, então um classificador de subobjetos para $\mathcal{E}$ é um $\mathcal{E}$-objeto $\Omega$ junto com um $\mathcal{E}$-morfismo

$$
\top: 1 \rightarrow \Omega
$$

tal que satisfaz a seguinte propriedade universal:

( $\Omega$-Axioma): Para cada monomorfismo $f: a \longmapsto b$ existe um único $\mathcal{E}$-morfismo $\chi_{f}$ : $d \rightarrow \Omega$, denominado o morfismo característico ou o caráter de $f$, tal que o diagrama

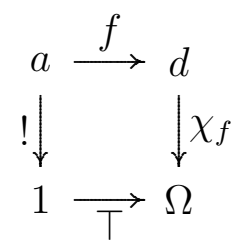


é um quadrado pullback.

Uma categoria $\mathcal{E}$ é dita um topos se e somente se $\mathcal{E}$ é finitamente bicompleta, possui classificador de subobjetos e exponenciação.

Diz-se que uma categoria $\mathcal{E}$ possui exponenciação se, para cada $\mathcal{E}$-objeto a, temos um funtor exponencial $(-)^{a}: \mathcal{E} \rightarrow \mathcal{E}$ que é adjunto à direita do funtor $(-) \times a$.

Em termos elementares, isso significa que $\mathcal{E}$ tem produto para cada par de $\mathcal{E}$-objetos e, para quaisquer $\mathcal{E}$-objetos dados $a$ e $b$, existe um $\mathcal{E}$-objeto, dito a exponencial de $b$ por $a$, denotado por $b^{a}$, e um $\mathcal{E}$-morfismo $e v_{a b}: b^{a} \times a \rightarrow b$, denominado morfismo avaliação, tal que vale a seguinte propriedade universal: Para todo $\mathcal{E}$-objeto $c$ e $\mathcal{E}$-morfismo $g: c \times a \rightarrow b$, existe um único $\mathcal{E}$-morfismo $\hat{g}: c \rightarrow b^{a}$ tal que o seguinte diagrama comuta:

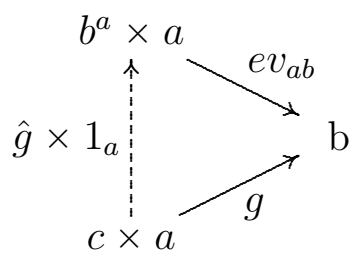

isto é, $e v_{a b} \circ\left(\hat{g} \times 1_{a}\right)=g$. Segue-se, imediatamente, que existe uma correspondência biunívoca entre as classes $\mathcal{E}(c \times a, b)$ e $\mathcal{E}\left(c, b^{a}\right)$. (Se $\mathcal{E}$ é uma categoria e $a$ e $b$ são dois $\mathcal{E}$-objetos, então $\mathcal{E}(a, b)$ denota a classe de todos os $\mathcal{E}$-morfismos de $a$ em $b$.) Quando uma categoria $\mathcal{E}$ é finitamente completa e possui exponenciação, então $\mathcal{E}$ é denominada uma categoria cartesiana fechada.

Dado que as categorias cartesianas fechadas possuem como consequência o 0-Axioma, segue-se que todo topos é uma categoria com morfismos verdade mas, no entanto, a conversa é falsa. Um exemplo de uma CTM que não é um topos é a categoria $S E T^{\leqslant \aleph_{0}}$, dos conjuntos no máximo enumeráveis e funções entre esses conjuntos, que satisfaz o 0-Axioma mas não possui exponenciação.

\section{Construções básicas em CTM's}

Vamos começar a examinar que construções e propriedades que são válidas em topos e que permanecem válidas nas categorias com morfismos verdade.

Seja $\mathcal{E}$, então, uma CTM.

ProposiÇÃo 1. Todo $\mathcal{E}$-morfismo do tipo $0 \stackrel{f}{\rightarrow}$ a é um monomorfismo.

Prova. De fato, suponha que existam morfismos $g, h: x \rightarrow 0$ tais que $f \circ g=f \circ h$. Pelo 0-Axioma, $g$ e $h$ são isomorfismos e, assim, $x$ é inicial em $\mathcal{E}$. Portanto, $g=h$ e $f$ é monomorfismo.

ProposiçÃo 2. Todo monomorfismo em $\mathcal{E}$ é um equalizador.

Prova. Seja $f: a \longmapsto b$ um monomorfismo em $\mathcal{E}$. Pelo $\Omega$-Axioma, temos o seguinte pullback:

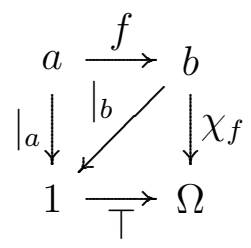

Queremos mostrar que $f$ é um equalizador de $\chi_{f}$ e $T_{b}:=\left.\top \circ\right|_{b}$. Verifiquemos primeiramente que $\chi_{f} \circ f=\top_{b} \circ f$. Como 1 é terminal em $\mathcal{E}$, temos que $\left.\right|_{a}=\left.\right|_{b} \circ f$ e, assim, 
$\chi_{f} \circ f=\left.\top \circ\right|_{a}=\left.\top \circ\right|_{b} \circ f=\top_{b} \circ f$.

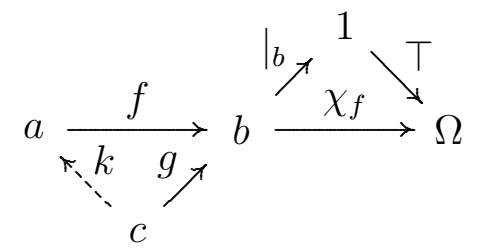

Seja, então, $g: c \rightarrow b$ tal que $\chi_{f} \circ g=\top_{b} \circ g$. (Ver diagrama acima.)

C

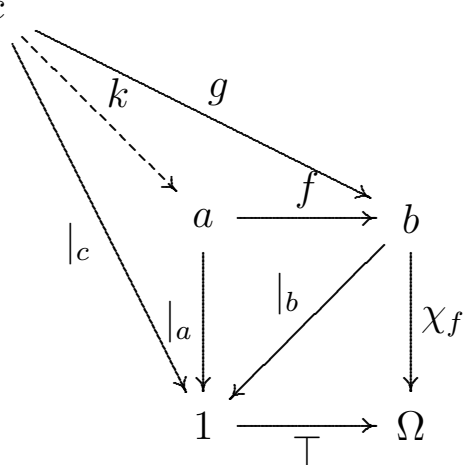

Como 1 é terminal, $\left.\right|_{c}=\left.\right|_{b} \circ g$. Assim, $\chi_{f} \circ g=\top_{b} \circ g=\left.\top \circ\right|_{b} \circ g=\left.\top \circ\right|_{c}$ e, portanto, o perímetro do diagrama acima comuta. Como se trata de um pullback, existe um único morfismo $k: c \rightarrow a$ tal que $f \circ k=g$. Assim, $f$ é um equalizador de $\chi_{f} \mathrm{e} \top_{b}$.

A proposição 2 possui dois corolários imediatos.

Corolário 3. Em $\mathcal{E}$, um morfismo é isomorfismo se e somente se ele é monomorfismo e epimorfismo.

Prova. De fato, vale em toda categoria que todo isomorfismo é monomorfismo e epimorfismo. Por outro lado, em $\mathcal{E}$, um morfismo que é epimorfismo e monomorfismo, pela proposição 2, é um epimorfismo equalizador. Mas, em uma categoria qualquer, um epimorfismo equalizador é sempre um isomorfismo.

Corolário 4. Em $\mathcal{E}$, um classificador de subobjetos $\top: 1 \rightarrow \Omega$ é um equalizador de $1_{\Omega} e T_{\Omega}$.

Prova. Considere o seguinte diagrama:

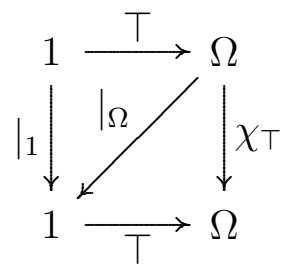

e o resultado é consequência imediata da proposição 2 , lembrando que $\chi_{\top}=1_{\Omega}$.

Vamos, agora, considerar a importante construção de imagens de morfismos com a correspondente demonstração que em CTM's todo morfismo possui uma decomposição epi-mono. É importante perceber que em nenhuma destas construções é necessário que a categoria possua exponenciação.

Considere a categoria com morfismos verdade $\mathcal{E}$ e seja $f: a \rightarrow b$ um $\mathcal{E}$-morfismo qualquer. Como $\mathcal{E}$ é finitamente cocompleta, forme o pushout:

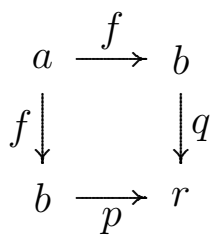


e seja $i m f: f(a) \longmapsto b$ o equalizador ${ }^{6}$ de $p$ e $q$. O morfismo $i m f$ é denominado o morfismo imagem de $f$. Como, pelo diagrama do pushout, $q \circ f=p \circ f$ e imf é um equalizador, existe um único $f^{*}$ tal que $f=i m f \circ f^{*}$. (Ver diagrama abaixo.)

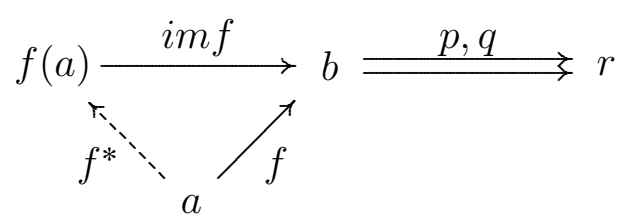

Vamos mostrar que $f^{*}$ é um epimorfismo e como equalizadores são monomorfismos, $f$ possui uma fatorização epi-mono. Para isto, vamos considerar primeiramente um resultado preliminar.

LEMA 5. No pushout acima de $f$ por $f$, se $p=q$, então $f$ é epimorfismo.

Prova. Considere os morfismos $g, h: b \rightarrow x$ tais que $g \circ f=h \circ f$.

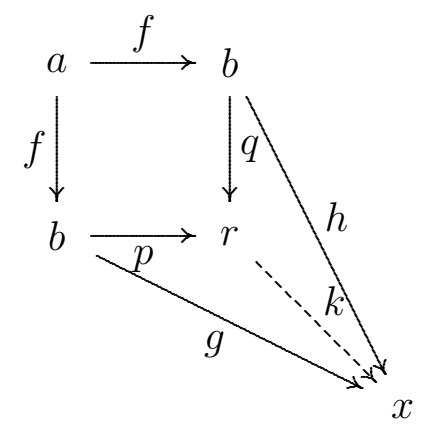

Como o quadrado é um pushout, existe um único $k$ tal que $k \circ p=g$ e $k \circ q=h$. Como $p=q$, temos: $g=k \circ p=k \circ q=h$ e, assim, $f$ é epimorfismo.

ProposiçÃo 6. Seja $f: a \rightarrow b$ um $\mathcal{E}$-morfismo. Assim, imf é o menor subobjeto de $b$ para o qual $f$ se fatora. Isto é, se há uma outra fatoração de $f$ dada por

$$
f=v \circ u: a \rightarrow c \longmapsto b
$$

para algum u e $v$ monomorfismo, então existe um único $k: f(a) \rightarrow$ c que faz o diagrama a seguir comutar

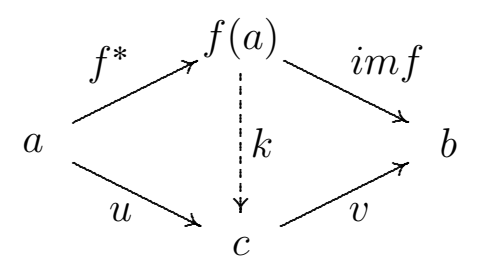

e, assim, imf está incluída em v.

Prova. Como $v$ é um monomorfismo, pela proposição $2, v$ é um equalizador de um par de morfismos $s, t: b \rightarrow d$. Considere o diagrama do pushout de $f$ por $f$ :

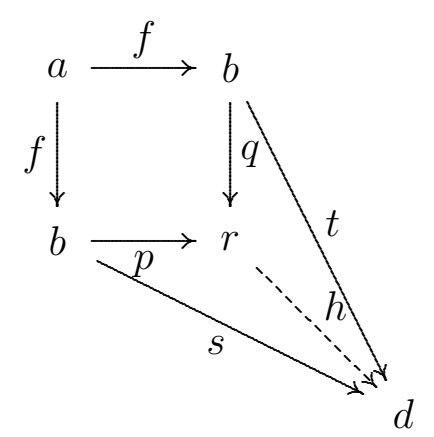

\footnotetext{
${ }^{6}$ Como limites e colimites de diagramas são sempre isomorfos, vamos usar o artigo definido fixando um equalizador específico qualquer. O mesmo abuso de linguagem será feito para todos os outros limites e colimites considerados no texto.
} 
Então, $s \circ f=s \circ v \circ u=t \circ v \circ u=t \circ f$. Assim, existe um único $h$ tal que $h \circ p=s$ e $h \circ q=t$.

Portanto, temos que:

$$
s \circ i m f=h \circ p \circ i m f=h \circ q \circ i m f=t \circ i m f .
$$

Como $v$ equaliza $s$ e $t$, temos:

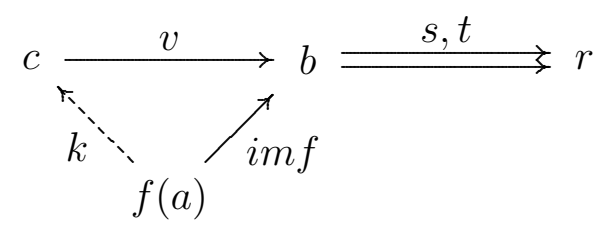

Logo, existe um único $k: f(a) \rightarrow c$ tal que $v \circ k=i m f$. Resta mostrar que $k \circ f^{*}=u$. Mas, $v \circ k \circ f^{*}=i m f \circ f^{*}=f=v \circ u$. Assim, $v \circ k \circ f^{*}=v \circ u$. Como $v$ é monomorfismo (cancelável à esquerda), segue-se que $k \circ f^{*}=u$, e temos o resultado.

Temos três corolários da proposição 6 .

Corolário 7. $f^{*}: a \rightarrow f(a)$ é um epimorfismo.

Prova. Aplicando o processo de construção de imagens no próprio $f^{*}$ temos:

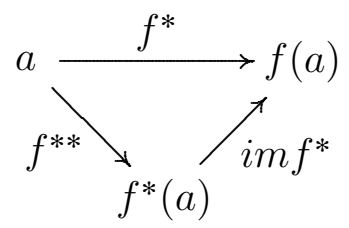

Fazendo $f^{*}=g$, temos o seguinte diagrama comutativo:

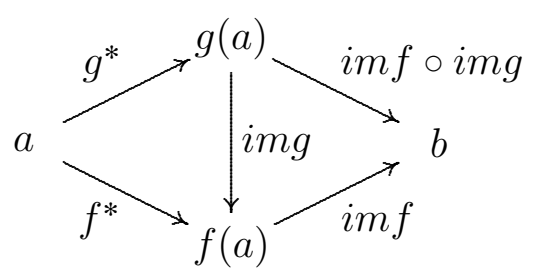

Então, imf o img é monomorfismo (composição de monomorfismos). Como imf é cancelável à esquerda, $i m g$ é o único morfismo que faz $i m f \circ i m g$ estar incluído em $i m f$. Por outro lado, aplicando a proposição 6 , temos que imf está incluído em $i m f \circ i m g$. Assim, $i m f \cong i m f \circ i m g$ e temos $g(a) \cong f(a)$.

Por definição, $i m g$ é o equalizador de:

$$
g(a) \stackrel{i m g}{\longrightarrow} f(a) \stackrel{p, q}{\longrightarrow} r
$$

em que $p$ e $q$ são dados pelo pushout:

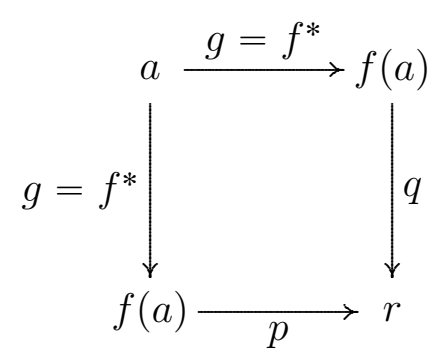

Como $p \circ i m g=q \circ i m g$ e $i m g$ é epimorfismo (pois é iso), então $p=q$. Segue-se, então, do lema 5 acima, que $f^{*}$ é um epimorfismo. 
Corolário 8. imfo $f^{*}: a \rightarrow f(a) \longmapsto b$ é uma fatoração epi-mono de $f$ que é única a menos de isomorfismos comutantes, isto é, se $v \circ u: a \rightarrow c \longmapsto b$ é tal que $f=v \circ u$, então existe exatamente um morfismo $k: f(a) \rightarrow c$ tal que o seguinte diagrama comuta:

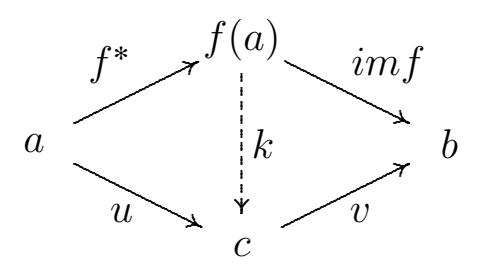

e $k$ é um isomorfismo.

Prova. Segue-se da proposição 6 que $k$ existe e é único. É preciso apenas mostrar que $k$ é isomorfismo. Como $v \circ k=i m f$ é monomorfismo, segue-se que $k$ é monomorfismo. Dualmente, como $k \circ f^{*}=u$ é epimorfismo, $k$ é epimorfismo também. Assim, $k$ é epi e mono e, pela proposição $3, k$ é um isomorfismo.

Corolário 9. $f: a \rightarrow b$ é epimorfismo se e somente se existe $g: f(a) \cong b$ tal que $g \circ f^{*}=f$.

Prova. Considere a fatoração de $f: a \rightarrow b$ dada por:

$$
i m f \circ f^{*}: a \rightarrow f(a) \longmapsto b .
$$

$(\Rightarrow)$ Se $f$ é epi, então imf é epi e como imf é mono, do corolário 3, temos que imf : $f(a) \cong b$.

$(\Leftarrow)$ Se tivermos

$$
g \circ f^{*}: a \rightarrow f(a) \cong b
$$

com $f=f^{*} \circ g$, então como $g$ é epi (pois é iso) e $f^{*}$ é epi, segue-se que $f$ é epimorfismo.

\section{Valores de verdade em CTM's}

A fim de construir semântica para linguagens proposicionais em categorias com morfismos verdade, precisamos primeiramente considerar como serão os valores de verdade neste tipo de categoria.

Se $a$ é um $\mathcal{E}$-objeto e 1 é terminal em $\mathcal{E}$, um morfismo do tipo $1 \rightarrow a$ é dito um elemento de $a$. Assim, considerando o classificador de subobjetos, morfismos do tipo $1 \rightarrow \Omega$ são os elementos de $\Omega$ denominados valores de verdade. O morfismo $\top: 1 \rightarrow \Omega$ é dito o valor de verdade verdadeiro. Vejamos como obtemos o valor de verdade correspondente ao falso.

Pela proposição 1 acima, o morfismo $0_{1}: 0 \longmapsto 1$ é um monomorfismo. Aplicando o $\Omega$-Axioma, obtemos o caráter de $0_{1}$ dado pelo pullback:

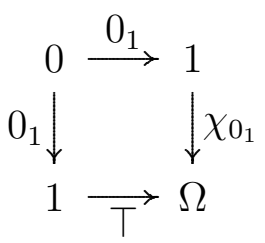

Definimos, então, o elemento de $\Omega$ dado por $\perp: 1 \rightarrow \Omega$, como $\perp:=\chi_{0_{1}}$. Denominamos o morfismo $\perp$ o valor de verdade falso.

Assim, temos o pullback dado por:

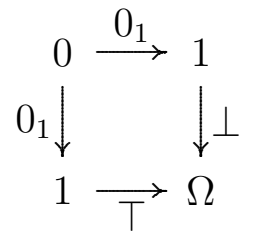


Vejamos o que acontece se $T=\perp$. Para isto, vamos considerar alguns fatos preliminares acerca de pullbacks que valem em categorias em geral.

FATO 10. Se o quadrado dado pelo diagrama

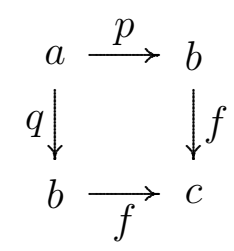

é um pullback, então p e q são epimorfismos.

Prova. Considere o diagrama abaixo lembrando que identidades são isomorfismos (mono e epi).

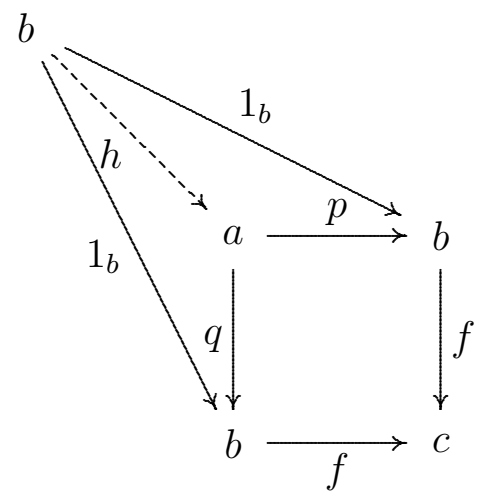

Como a parte periférica do diagrama comuta, então existe um único $h: b \rightarrow a$ tal que $p \circ h=1_{b}$ e $q \circ h=1_{b}$. Como $1_{b}$ é epi, $p$ e $q$ devem ser epimorfismos.

FATo 11. Se o quadrado dado pelo diagrama

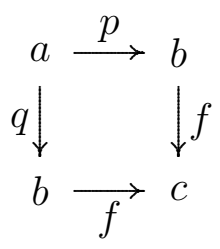

é um pullback, então f é monomorfismo se e somente se $p=q$.

Prova. Suponha que $f$ é monomorfismo. Como $f \circ p=f \circ q$ e $f$ é cancelável à esquerda, segue-se que $p=q$.

Suponha, agora, que $p=q$ e considere o diagrama

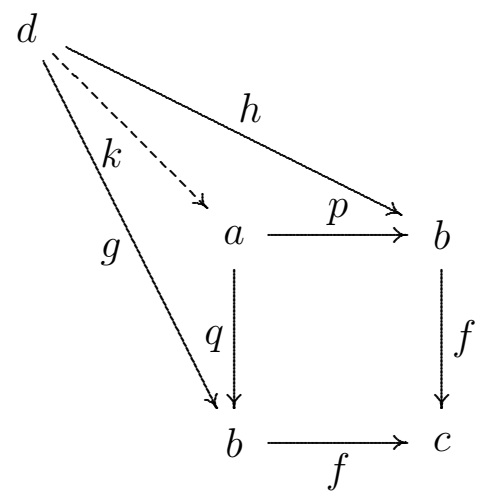

com $f \circ h=f \circ g$. Então, existe um único $k$ tal que $h=p \circ k$ e $g=q \circ k$. Como $p=q$, segue-se que $g=h$, isto é, $f$ é monomorfismo. 
FATO 12. Se o quadrado dado pelo diagrama

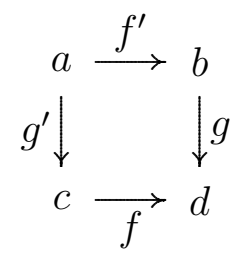

é um pullback e g é monomorfismo, então g' também é monomorfismo.

Prova. Considere os morfismos $u, v: e \rightarrow a$ tais que $g^{\prime} \circ u=g^{\prime} \circ v$. Queremos mostrar que $u=v$. Faça $g^{\prime \prime}=g^{\prime} \circ u$ e $f^{\prime \prime}=f^{\prime} \circ u$ (ver diagrama).

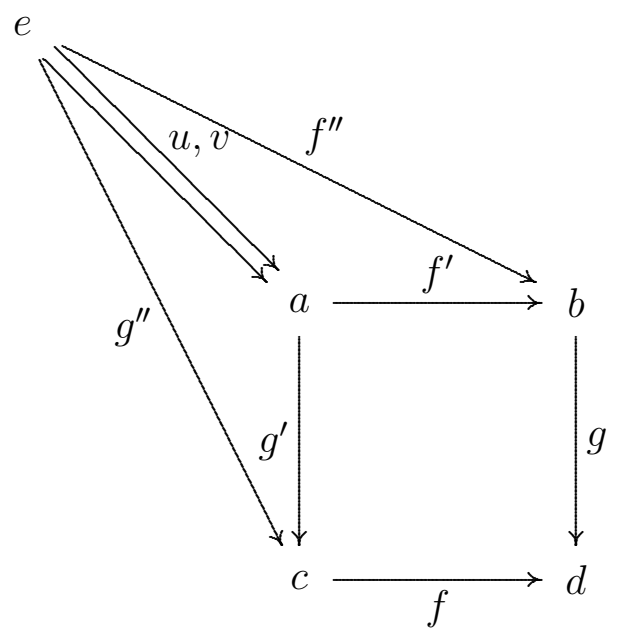

Temos, então, que

$$
\begin{aligned}
f \circ g^{\prime \prime} & =f \circ g^{\prime} \circ u & & \text { pois } g^{\prime \prime}=g^{\prime} \circ u \\
& =g \circ f^{\prime} \circ u & & \text { pois } g \circ f^{\prime}=f \circ g^{\prime} \\
& =g \circ f^{\prime \prime} & & \text { pois } f^{\prime \prime}=f^{\prime} \circ u
\end{aligned}
$$

Como o quadrado é um pullback, $u$ é o único morfismo tal que $g^{\prime \prime}=g^{\prime} \circ u$ e $f^{\prime \prime}=f^{\prime} \circ u$. Como $g^{\prime} \circ u=g^{\prime} \circ v$, temos também que $g^{\prime \prime}=g^{\prime} \circ v$. Segue-se, então, que

$$
\begin{aligned}
g \circ f^{\prime} \circ v & =f \circ g^{\prime} \circ v & & \text { pois } g \circ f^{\prime}=f \circ g^{\prime} \\
& =f \circ g^{\prime} \circ u & & \text { pois } g^{\prime} \circ u=g^{\prime} \circ v \\
& =f \circ g^{\prime \prime} & & \text { pois } g^{\prime} \circ u=g^{\prime \prime} \\
& =g \circ f^{\prime \prime} & & \text { estabelecido acima }
\end{aligned}
$$

Como $g$ é monomorfismo (cancelável à esquerda), temos $f^{\prime \prime}=f^{\prime} \circ v$. Assim, $v$ também fatora ambos $g^{\prime \prime}$ e $f^{\prime \prime}$. Como $u$ é única, temos que $u=v$. Logo, $g^{\prime}$ é monomorfismo.

Voltemos, agora, para o ambiente de uma categoria com morfismos verdade $\mathcal{E}$. Os fatos estabelecidos acima implicam o seguinte resultado.

Lema 13. Em $\mathcal{E}$, se o quadrado dado pelo diagrama

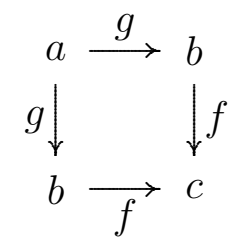

é um pullback, então $a \cong b$, isto é, a e b são objetos isomorfos.

Prova. Pelo fato 10, $g$ é epimorfismo. Pelo fato 11, $f$ é monomorfismo. Assim, pelo fato $12, g$ também é monomorfismo. Como $\mathcal{E}$ é uma categoria com morfismos verdade, segue-se do corolário 3 que $g$ é isomorfismo. Logo, $a \cong b$. 
Segue-se, então, uma propriedade que estabelece uma relação entre os valores de verdade verdadeiro e falso.

ProposiçÃo 14. Em uma categoria com morfismos verdade não degenerada, temos que $\top \neq \perp$.

Prova. O morfismo falso é definido pelo pullback

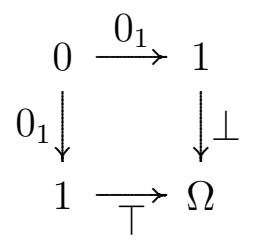

Suponha que $T=\perp$. Pelo lema 13 , temos que $0 \cong 1$, mas, nesse caso, a categoria é degenerada.

Segue-se assim, que se $T=\perp$, então $\mathcal{E}$ é uma categoria degenerada. (Todos os objetos são isomorfos entre si.)

\section{Morfismos verdade em CTM's}

Considere uma categoria com morfismos verdade $\mathcal{E}$. O classificador de subobjetos $\Omega$ é um $\mathcal{E}$-objeto cujos elemento (morfismos do tipo $1 \rightarrow \Omega$ ) são denominados valores de verdade. Se $\mathcal{E}$ é não degenerada, temos pelo menos dois elementos em $\Omega$, os morfismos $\top, \perp: 1 \rightarrow \Omega$. Queremos, agora, definir certos morfismos em $\mathcal{E}$ que correponderão às funções de verdade associadas aos conectivos de negação, conjunção, implicação e disjunção das linguagens proposicionais usuais.

Se $a$ é um $\mathcal{E}$-objeto e $n$ é um inteiro positivo, vamos denotar por $a^{n}$ o produto (categorial) de $n$ cópias de $a$, isto é:

$$
a^{n}:=\underbrace{a \times a \times \ldots \times a}_{n \text { vezes }} .
$$

Assim, um morfismo verdade em $\mathcal{E}$ é qualquer morfismo do tipo:

$$
\Omega^{n} \rightarrow \Omega
$$

para algum inteiro positivo $n$. Como de costume, o número $n$ é dito a aridade do morfismo verdade. Estamos interessados principalmente nos casos em que $n$ é igual a 1 ou 2.

Suponhamos, por exemplo que temos um morfismo binário (de aridade 2)

$$
f: \Omega \times \Omega \rightarrow \Omega .
$$

Considere, então, dois valores de verdade (elementos de $\Omega$ ) quaisquer $x, y: 1 \rightarrow \Omega$. Tomando o produto $\langle x, y\rangle$ dado pelo diagrama

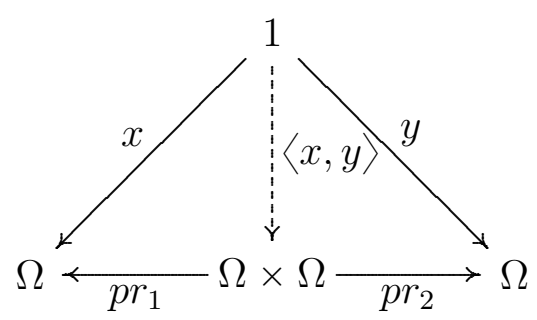


e, compondo com o morfismo $f$ obtemos (ver diagrama)

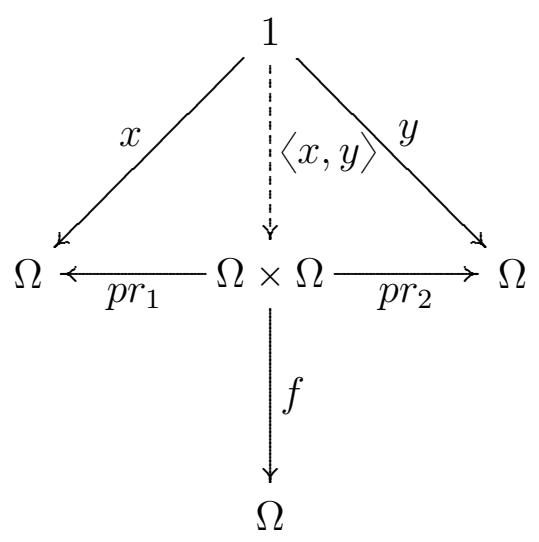

um morfismo $f \circ\langle x, y\rangle: 1 \rightarrow \Omega$, que é também um elemento de $\Omega$ (um valor de verdade).

Assim, dado um morfismo verdade $f$ qualquer, sempre podemos avaliar o resultado de compor $f$ com morfismos produto dos valores de verdade $T \mathrm{e} \perp$.

No que se segue, vamos mostrar como construir os morfismos verdade no ambiente das CTM's. O que justifica sua qualificação como categorias com morfismos verdade. Além disso, vamos estudar o comportamento desses morfismos com respeito aos valores de verdade $T$ e $\perp$. Esse estudo justificará denominar tais morfismos como morfismo negação, conjunção, implicação e disjunção.

5.1. Morfismo negação. Como 1 é terminal em $\mathcal{E}$, os valores de verdade são sempre monomorfismos. Considere, então, o monomorfismo $\perp: 1 \longmapsto \Omega$ que é o falso. Aplicando o $\Omega$-Axioma a $\perp$, temos o seguinte pullback:

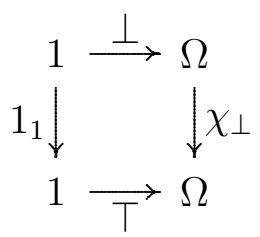

Definimos, então, o morfismo verdade $f_{\neg}: \Omega \rightarrow \Omega$ como o caráter de $\perp$, isto é, $f_{\neg}:=\chi_{\perp}$. Tal morfismo verdade é dito o morfismo negação.

5.2. Morfismo conjunção. Considere o produto $\Omega \times \Omega$ e construa o morfismo produto $\langle\top, T\rangle$ dado pelo diagrama:

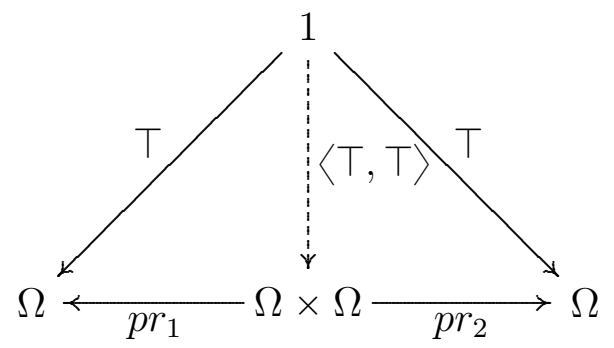

Novamente, como 1 é terminal, $\langle\top, T\rangle: 1 \longmapsto \Omega \times \Omega$ é um monomorfismo. Aplicando o $\Omega$-Axioma a $\langle T, T\rangle$, temos o seguinte pullback:

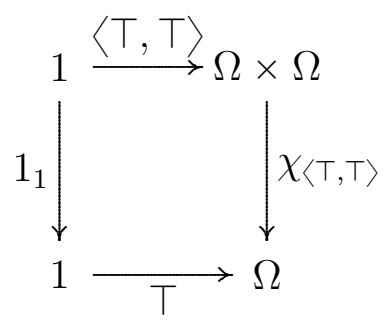


Definimos, então, o morfismo verdade $f_{\wedge}: \Omega \times \Omega \rightarrow \Omega$ como o caráter de $\langle\top, T\rangle$, isto é, $f_{\wedge}:=\chi_{\langle T, T\rangle}$. Tal morfismo verdade é dito o morfismo conjunção.

5.3. Morfismo implicação. Considere, agora, o par de morfismos verdade dados por:

$$
f_{\wedge}, p r_{1}: \Omega \times \Omega \rightarrow \Omega .
$$

o morfismo conjunção e a primeira projeção do produto. (Note que as projeções, nesse caso, também são morfismos verdade.)

Tome um equalizador desse par de morfismos.

$$
(\leqslant) \stackrel{e}{\longrightarrow} \Omega \times \Omega \stackrel{f_{\wedge}, p r_{1}}{\longrightarrow} \Omega
$$

Como todo equalizador é monomorfismo, aplicando o $\Omega$-Axioma a $e:(\leqslant) \longmapsto \Omega \times \Omega$, temos o seguinte pullback:

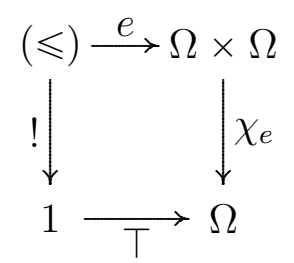

Definimos, então, o morfismo verdade $f_{\supset}: \Omega \times \Omega \rightarrow \Omega$ como o caráter de $e$, um equalizador de $f_{\wedge}$ e $p r_{1}$, isto é, $f_{\wedge}:=\chi_{e}$. Tal morfismo verdade é dito o morfismo implicação.

5.4. Morfismo disjunção. Considere o morfismo verdade $T_{\Omega}: \Omega \rightarrow \Omega$ definido como $T_{\Omega}:=\left.\top_{\circ} \circ\right|_{\Omega}: \Omega \rightarrow 1 \rightarrow \Omega$. tome o morfismo produto $\left\langle T_{\Omega}, 1_{\Omega}\right\rangle: \Omega \rightarrow \Omega \times \Omega$ dado pelo diagrama

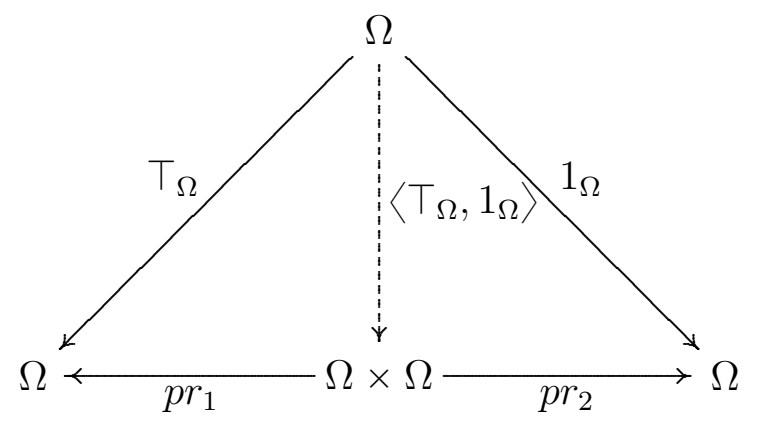

Tome, também o produto invertido $\left\langle 1_{\Omega}, \top_{\Omega}\right\rangle: \Omega \rightarrow \Omega \times \Omega$. Forme, agora, o coproduto

$$
\left[\left\langle T_{\Omega}, 1_{\Omega}\right\rangle,\left\langle 1_{\Omega}, T_{\Omega}\right\rangle\right]: \Omega+\Omega \rightarrow \Omega \times \Omega
$$

dado pelo diagrama:

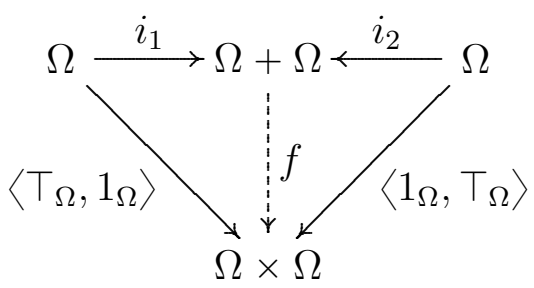

$\operatorname{com} f:=\left[\left\langle\top_{\Omega}, 1_{\Omega}\right\rangle,\left\langle 1_{\Omega}, T_{\Omega}\right\rangle\right]$

Aplicando o processo de construção de imagens de morfismos a $f$, obtemos um morfismo

$$
f(\Omega+\Omega) \stackrel{i m f}{\longrightarrow} \Omega \times \Omega,
$$

(que é um equalizador do pushout de $f$ por $f$ ). 
Como imf é monomorfismo, aplicando o $\Omega$-Axioma a imf : $f(\Omega+\Omega) \longmapsto \Omega \times \Omega$, temos o seguinte pullback:

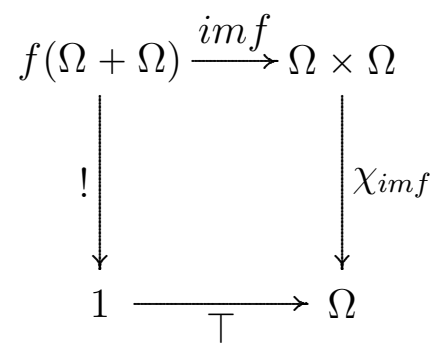

Definimos, então, o morfismo verdade $f_{\vee}: \Omega \times \Omega \rightarrow \Omega$ como o caráter da imagem do morfismo coproduto $\left[\left\langle T_{\Omega}, 1_{\Omega}\right\rangle,\left\langle 1_{\Omega}, T_{\Omega}\right\rangle\right]$, isto é, $f_{\vee}:=\chi_{f}$. Tal morfismo verdade é dito o morfismo disjunção.

5.5. Resumindo. Vamos resumir as definições dos morfismos verdade construídos até o momento.

- $f_{\neg}: \Omega \rightarrow \Omega$ é o caráter de $\perp: 1 \rightarrow \Omega$;

- $f_{\wedge}: \Omega \times \Omega \rightarrow \Omega$ é o caráter do morfismo produto $\langle\top, T\rangle: 1 \rightarrow \Omega \times \Omega$;

- $f_{\supset}: \Omega \times \Omega \rightarrow \Omega$ é o caráter equalizador do par de morfismos $f_{\wedge}, p r_{1}: \Omega \times \Omega \rightarrow \Omega$;

- $f_{\vee}: \Omega \times \Omega \rightarrow \Omega$ é o caráter da imagem do morfismo coproduto $\left[\left\langle T_{\Omega}, 1_{\Omega}\right\rangle,\left\langle 1_{\Omega}, T_{\Omega}\right\rangle\right]$ : $\Omega+\Omega \rightarrow \Omega \times \Omega$.

É importante, nesse ponto, observar que todas estas construções apresentadas podem ser executadas em uma categoria com morfismos verdade. (Tais categorias poderiam ser denominadas categorias proposicionais.) Note que, em nenhum passo dessas construções, foi necessário que a categoria tivesse exponenciação. Não é preciso operar em um ambiente de topos se estamos interessados apenas em linguagens proposicionais. A única consequência da existência de exponenciação que precisamos é o que denominamos 0Axioma: todo morfismo cujo codomínio é um objeto inicial é um isomorfismo. Segue-se desse axioma que morfismos do tipo $a \rightarrow 0$ são monomorfismos, e isso foi necessário para definir o valor de verdade falso; e em seguida o morfismo negação. Todo o resto é obtido por meio de limites, colimites, imagens e o $\Omega$-Axioma, cujas construções estão disponíveis em CTM's.

Nosso próximo passo é estudar o comportamento dos morfismos verdade com respeito aos valores de verdade $\top$ e $\perp$, justificando, assim, a denominção dos mesmos. É esse estudo que faremos agora.

\section{Morfismos verdade e valores de verdade}

Vamos, agora, investigar o comportamento dos morfismos verdade com respeito aos valores de verdade verdadeiro e falso.

6.1. Tabela do morfismo negação. Observe que, da comutatividade do diagrama pullback que define $f_{\neg}$,

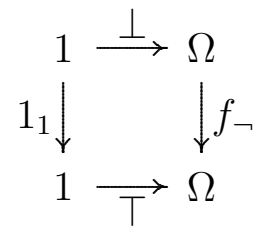

segue-se que $f_{\neg} \circ \perp=\top \circ 1_{1}=\top$. 
Por outro lado, considere o diagrama:

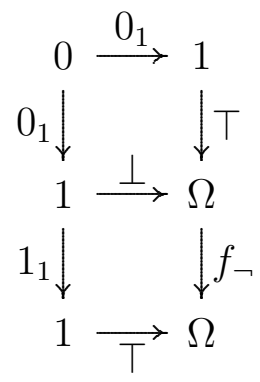

O quadrado superior é o pullback (invertido) que define $\perp$. O quadrado inferior é o pullback que define $f \neg$. Logo, pelo lema do Pullback, o retângulo externo é um pullback. Assim, pelo $\Omega$-Axioma, temos $f_{\neg} \circ \top=\chi_{0_{1}}=\perp$.

Podemos, então, resumir o comportamento (composição) do morfismo negação $f_{\neg}$ com respeito aos valores de verdade $T, \perp: 1 \rightarrow \Omega$ na seguinte tabela:

\begin{tabular}{c|c}
$x$ & $f_{\neg} \circ x$ \\
\hline$\top$ & $\perp$ \\
$\perp$ & $\top$
\end{tabular}

que corresponde à tabela usual da negação da lógica proposicional clássica.

6.2. Tabela do morfismo conjunção. Novamente, pela comutatividade do diagrama

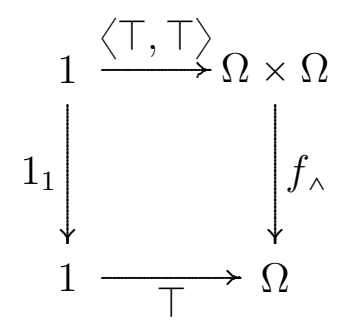

segue-se que $f_{\wedge} \circ\langle T, T\rangle=T \circ 1_{1}=T$.

Considere, agora, o seguinte diagrama:

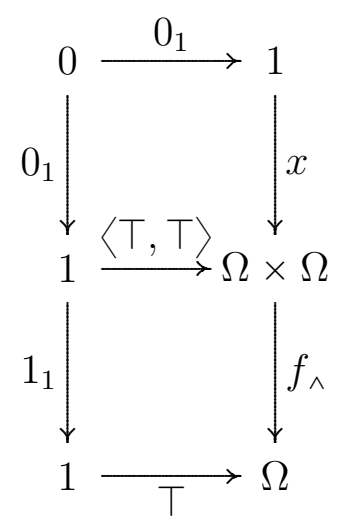

em que $x$ é $\langle\top, \perp\rangle,\langle\perp, \top\rangle$ ou $\langle\perp, \perp\rangle$.

O quadradro inferior do diagrama acima é o pullback que define $f_{\wedge}$. Vamos mostrar que o quadrado superior é um pullback para qualquer das escolhas de $x$. Seguirá daí, pelo Lema do Pullback, que o retângulo externo é um pullback. Então, pelo $\Omega$-Axioma, teremos que $f_{\wedge} \circ x=\chi_{0_{1}=\perp}$. 
Considere, então, o diagrama superior

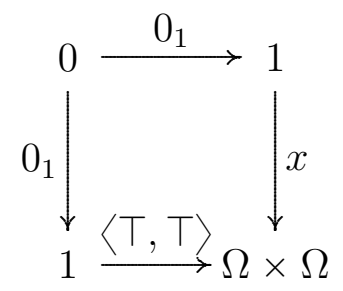

Como 0 é inicial, o quadrado é comutativo. Seja, então, $g: a \rightarrow 1$ tal que $\langle\top, \top\rangle \circ g=x \circ g$.

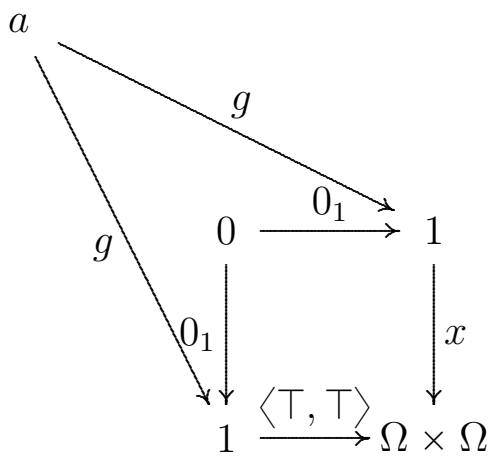

Temos três casos:

(1) Se $x=\langle\top, \perp\rangle$, então $\langle\top, T\rangle \circ g=\langle\top, \perp\rangle \circ g$ e temos $\langle\top \circ g, \top \circ g\rangle=\langle\top \circ g, \perp \circ g\rangle$, obtendo $\top \circ g=\perp \circ g$.

(2) Se $x=\langle\perp, \top\rangle$, então um raciocínio análogo resulta que $\top \circ g=\perp \circ g$.

(3) Se $x=\langle\perp, \perp\rangle$, então também segue que $\top \circ g=\perp \circ g$.

Portanto, em qualquer caso temos que $\top \circ g=\perp \circ g$. Tome, agora, o pullback que define o valor de verdade $\perp$.

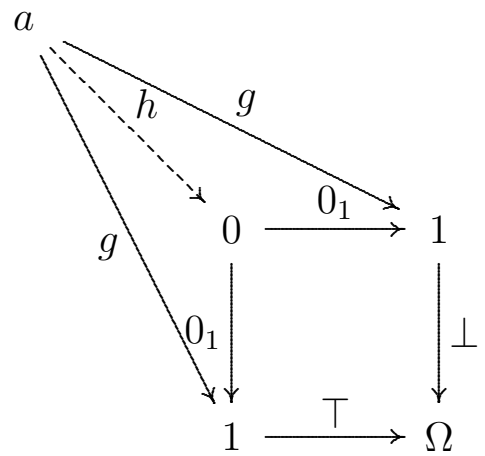

Como o quadrado é um pullback e $T \circ g=\perp \circ g$, segue-se que existe um único $h: a \rightarrow 0$. (A comutatividade dos triângulos é óbvia, pois 1 é terminal em $\mathcal{E}$.) Pelo 0-Axioma, tem-se que $h$ é iso e, assim, $a$ é inicial em $\mathcal{E}$. Transortando $h$ para nosso diagrama inicial temos:

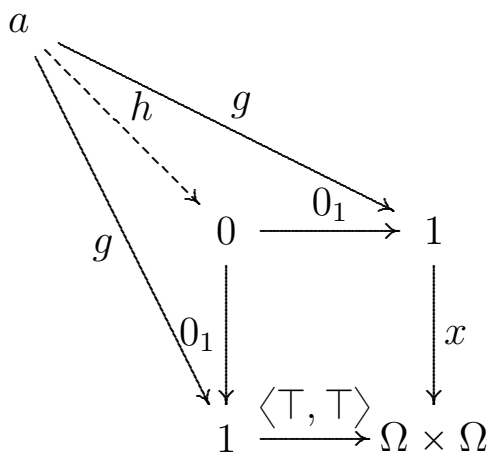

Como 1 é terminal, $0_{1} \circ h=g$ e, assim, o quadrado é um pullback. Segue-se, então, que

$$
f_{\wedge} \circ\langle\top, \perp\rangle=f_{\wedge} \circ\langle\perp, \top\rangle=f_{\wedge} \circ\langle\perp, \perp\rangle=\perp .
$$


Podemos, então, resumir o comportamento (composição) do morfismo conjunção $f_{\wedge}$ com respeito aos valores de verdade $\top, \perp: 1 \rightarrow \Omega$ na seguinte tabela:

\begin{tabular}{cc|c}
$x$ & $y$ & $f_{\wedge} \circ\langle x, y\rangle$ \\
\hline$\top$ & $\top$ & $\top$ \\
$\top$ & $\perp$ & $\perp$ \\
$\perp$ & $\top$ & $\perp$ \\
$\perp$ & $\perp$ & $\perp$
\end{tabular}

que corresponde à tabela usual da conjunção da lógica proposicional clássica.

6.3. Tabela do morfismo implicação. Considere o seguinte diagrama:

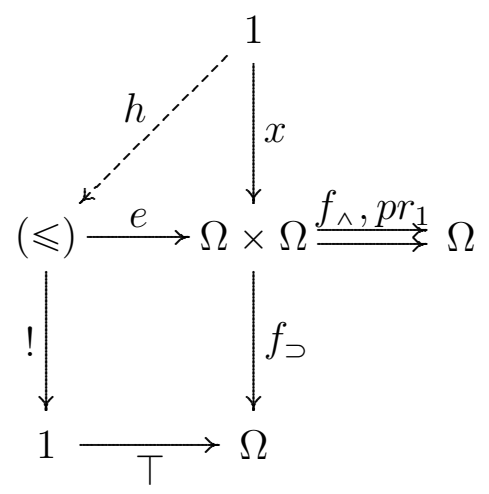

em que $x$ é $\langle\top, T\rangle,\langle\perp, T\rangle$ ou $\langle\perp, \perp\rangle$. Nesse caso, para qualquer escolha de $x$, usando a tabela para o morfismo conjunção e as propriedades da projeção $p r_{1}$, temos que:

$$
f_{\wedge} \circ x=p r_{1} \circ x .
$$

Como $e$ é um equalizador de $f_{\wedge}$ e $p r_{1}$, existe um único morfismo $h: 1 \rightarrow(\leqslant)$ tal que $e \circ h=x$. Mas, então, como o quadrado é o pullback que define $f_{\supset}$, temos, por comutatividade, que $T \circ !=f_{\supset} \circ$ e. Assim,

$$
\begin{aligned}
f_{\supset} \circ x & =f_{\supset} \circ e \circ h \\
& =\top \circ ! \circ h \\
& =\top \circ 1_{1} \\
& =\top
\end{aligned}
$$

Segue-se, então, que:

$$
f_{\supset} \circ\langle\top, \top\rangle=f_{\supset} \circ\langle\perp, \top\rangle=f_{\supset} \circ\langle\perp, \perp\rangle=\top .
$$

Resta calcular o valor de verdade de $f_{\supset}$ aplicado ao morfismo produto $\langle T, \perp\rangle$. Para isso, considere o diagrama:

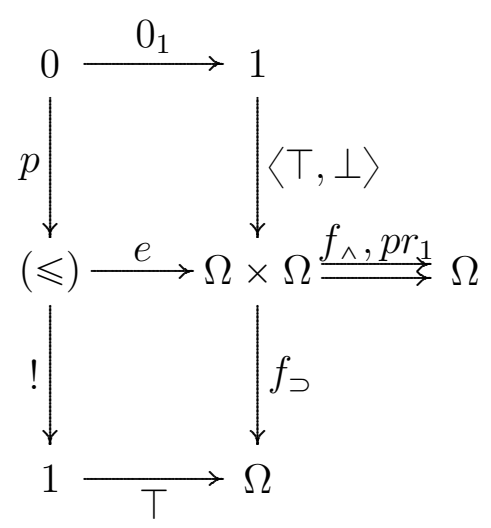


O quadrado inferior é o pullback que define $f_{\supset}$. Mostremos que o quadrado superior é um pullback. Seguirá daí que o retângulo externo é um pullback e, pelo $\Omega$-Axioma, teremos que

$$
f_{\supset} \circ\langle\top, \perp\rangle=\chi_{o_{1}}=\perp
$$

obtendo o que desejamos.

Examinemos, então, o quadrado superior:

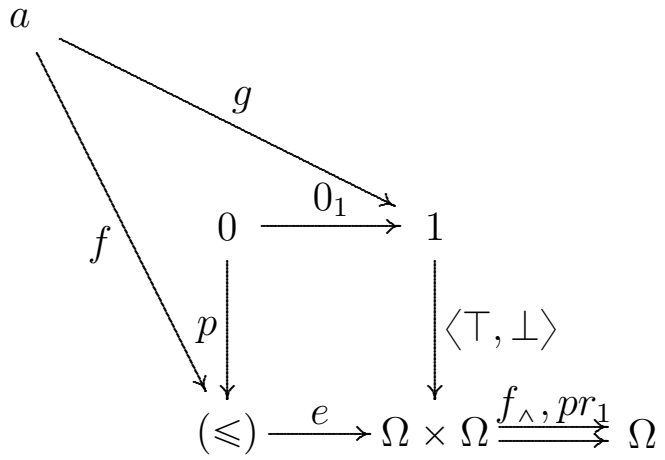

Como 0 é inicial, o quadrado é comutativo. Considere os morfismos $f: a \rightarrow(\leqslant)$ e $g: a \rightarrow 1$. Suponha que $e \circ f=\langle\top, \perp\rangle \circ g$. Como $e$ equaliza $f_{\wedge}$ e $p r_{1}$, temos que $f_{\wedge} \circ e=p r_{1} \circ e$. Compondo com $f$ temos:

$$
f_{\wedge} \circ e \circ f=p r_{1} \circ e \circ f .
$$

Mas, por um lado,

$$
f_{\wedge} \circ e \circ f=f_{\wedge} \circ\langle\top, \perp\rangle \circ g=\perp \circ g .
$$

(A última igualdade segue da aplicação da tabela para o morfismo conjunção.)

Por outro lado,

$$
p r_{1} \circ e \circ f=p r_{1} \circ\langle\top, \perp\rangle \circ g=\top \circ g
$$

(Aplicando a propriedade da projeção.)

Portanto, temos que $\top \circ g=\perp \circ g$. Agora, por um argumento análogo ao caso do morfismo conjunção, temos que o quadrado acima é um pullback. Logo, segue-se que $f_{\supset} \circ\langle\top, \perp\rangle=\perp$, como queríamos.

Podemos, então, resumir o comportamento (composição) do morfismo implicação $f_{\supset}$ com respeito aos valores de verdade $T, \perp: 1 \rightarrow \Omega$ na seguinte tabela:

\begin{tabular}{cc|c}
$x$ & $y$ & $f_{\supset} \circ\langle x, y\rangle$ \\
\hline$\top$ & $\top$ & $\top$ \\
$\top$ & $\perp$ & $\perp$ \\
$\perp$ & $\top$ & $\top$ \\
$\perp$ & $\perp$ & $\top$
\end{tabular}

que corresponde à tabela usual da implicação material da lógica proposicional clássica.

6.4. Tabela do morfismo disjunção. Observemos, primeiramente que, do diagrama

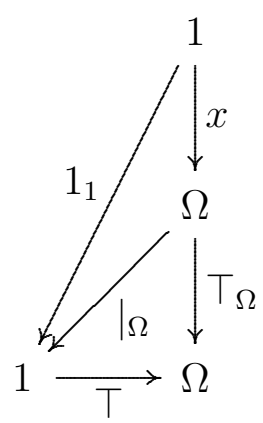


temos: $T_{\Omega} \circ x=\left.\top \circ\right|_{\Omega} \circ x=\top \circ 1_{1}=\top$. (Assim, a composição de $T_{\Omega}$ com qualquer valor de verdade resulta em $\top$.)

Segue-se, então, que $T_{\Omega} \circ T=T_{\Omega} \circ \perp=T$.

O processo de construção de $i m\left[\left\langle\top_{\Omega}, 1_{\Omega}\right\rangle,\left\langle 1_{\Omega}, \top_{\Omega}\right\rangle\right]$ provém do pushout dado por:

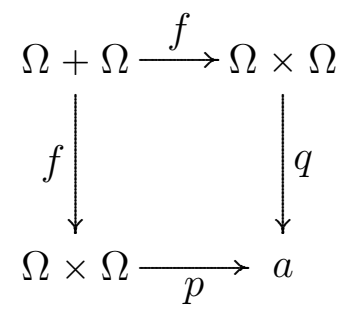

em que $f:=\left[\left\langle T_{\Omega}, 1_{\Omega}\right\rangle,\left\langle 1_{\Omega}, T_{\Omega}\right\rangle\right]$

Então, temos que:

$$
p \circ\left[\left\langle\top_{\Omega}, 1_{\Omega}\right\rangle,\left\langle 1_{\Omega}, \top_{\Omega}\right\rangle\right]=q \circ\left[\left\langle\top_{\Omega}, 1_{\Omega}\right\rangle,\left\langle 1_{\Omega}, \top_{\Omega}\right\rangle\right],
$$

Isto é,

$$
\left[p \circ\left\langle\top_{\Omega}, 1_{\Omega}\right\rangle, p \circ\left\langle 1_{\Omega}, \top_{\Omega}\right\rangle\right]=\left[q \circ\left\langle\top_{\Omega}, 1_{\Omega}\right\rangle, q \circ\left\langle 1_{\Omega}, \top_{\Omega}\right\rangle\right] .
$$

Temos, então, duas equações:

$$
\begin{aligned}
& p \circ\left\langle\top_{\Omega}, 1_{\Omega}\right\rangle=q \circ\left\langle\top_{\Omega}, 1_{\Omega}\right\rangle \\
& p \circ\left\langle 1_{\Omega}, \top_{\Omega}\right\rangle=q \circ\left\langle 1_{\Omega}, \top_{\Omega}\right\rangle
\end{aligned}
$$

Compondo a equação $(I)$ com $\top$, e usando a propriedade do início da seção, temos:

$$
\begin{array}{ll}
p \circ\left\langle T_{\Omega}, 1_{\Omega}\right\rangle \circ \top & =q \circ\left\langle T_{\Omega}, 1_{\Omega}\right\rangle \circ \top \\
p \circ\left\langle T_{\Omega} \circ \top, 1_{\Omega} \circ \top\right\rangle & =q \circ\left\langle T_{\Omega} \circ \top, 1_{\Omega} \circ \top\right\rangle \\
p \circ\langle\top, T\rangle & =q \circ\langle\top, T\rangle
\end{array}
$$

Compondo a equação $(I)$ com $\perp$ temos:

$$
\begin{array}{ll}
p \circ\left\langle\top_{\Omega}, 1_{\Omega}\right\rangle \circ \perp & =q \circ\left\langle T_{\Omega}, 1_{\Omega}\right\rangle \circ \perp \\
p \circ\left\langle\top_{\Omega} \circ \perp, 1_{\Omega} \circ \perp\right\rangle & =q \circ\left\langle\top_{\Omega} \circ \perp, 1_{\Omega} \circ \perp\right\rangle \\
p \circ\langle\top, \perp\rangle & =q \circ\langle\top, \perp\rangle
\end{array}
$$

Compondo a equação $(I I)$ com $\perp$ temos:

$$
\begin{array}{ll}
p \circ\left\langle 1_{\Omega}, \top_{\Omega}\right\rangle \circ \perp & =q \circ\left\langle 1_{\Omega}, \top_{\Omega}\right\rangle \circ \perp \\
p \circ\left\langle 1_{\Omega} \circ \perp, \top_{\Omega} \circ \perp\right\rangle & =q \circ\left\langle 1_{\Omega} \circ \perp, \top_{\Omega} \circ \perp\right\rangle \\
p \circ\langle\perp, \top\rangle & =q \circ\langle\perp, \top\rangle
\end{array}
$$

Assim, se $x: 1 \rightarrow \Omega \times \Omega$ for $\langle\top, \top\rangle,\langle\top, \perp\rangle$ ou $\langle\perp, \top\rangle$, segue-se que $p \circ x=q \circ x$. Considere, então, o seguinte diagrama em que $f:=\left[\left\langle\top_{\Omega}, 1_{\Omega}\right\rangle,\left\langle 1_{\Omega}, \top_{\Omega}\right\rangle\right]$ e $x$ é como acima:

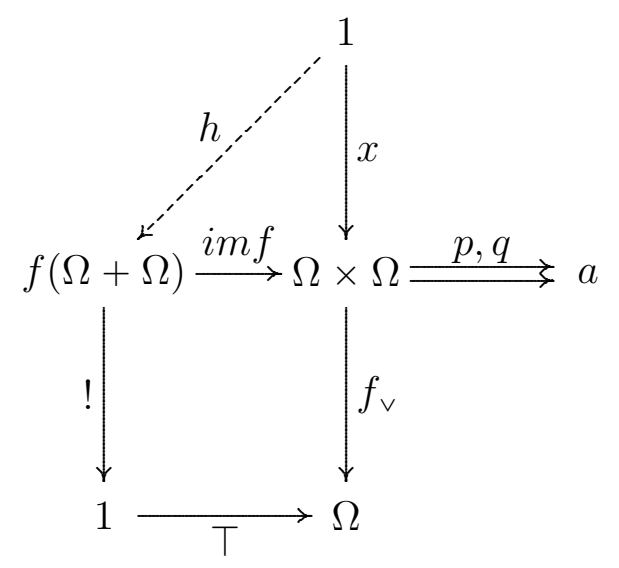


Como imf é equalizador de $p$ e $q$ e, além disso, $p \circ x=q \circ x$, segue-se que existe um único $h: 1 \rightarrow f(\Omega+\Omega)$ tal que $i m f \circ h=x$. Como o quadrado comuta, temos

$$
f_{\vee} \circ i m f=\top \circ !
$$

Compondo com $h$, temos:

$$
f_{\vee} \circ \underbrace{i m f \circ h}_{x}=\top \circ \underbrace{! \circ h}_{1_{1}} .
$$

Portanto, $f_{\vee} \circ x=\top$.

Assim, segue-se que $f_{\vee} \circ\langle\top, T\rangle=f_{\vee} \circ\langle\perp, T\rangle=f_{\vee} \circ\langle\perp, \perp\rangle=\top$.

Até o momento, o comportamento do morfismo $f_{\vee}$ com respeito aos valores de verdade podem ser resumidos de acordo com a seguinte tabela:

\begin{tabular}{cc|c}
$x$ & $y$ & $f_{\vee} \circ\langle x, y\rangle$ \\
\hline$\top$ & $\top$ & $\top$ \\
$\top$ & $\perp$ & $\top$ \\
$\perp$ & $\top$ & $\top$ \\
$\perp$ & $\perp$ & $?$
\end{tabular}

Resta mostrar que $f_{\vee} \circ\langle\perp, \perp\rangle=\perp$. Mas, nesse ponto, parece que temos um problema. Seguindo os métodos de prova dos casos anteriores, parece que a estratégia a ser adotada é a seguinte:

Consideremos o diagrama

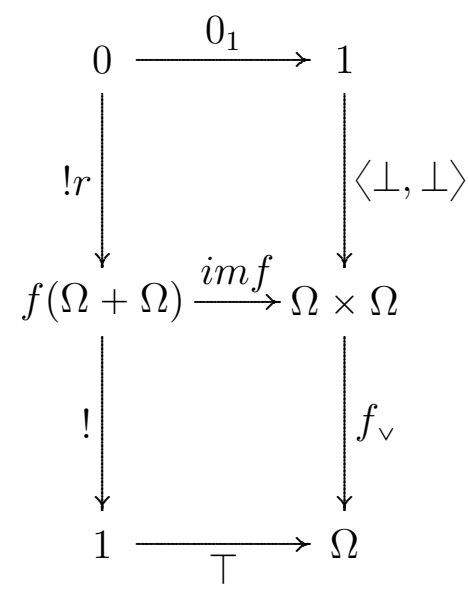

Como o quadrado inferior é o pullback que define $f_{\vee}$, se mostrarmos que o quadrado superior é um pullback, então, pelo Lema do Pullback, o retângulo exterior é um pullback e, pelo $\Omega$-Axioma, temos que $f_{\vee} \circ\langle\perp, \perp\rangle=\chi_{0_{1}}=\perp$, estabelecendo o caso que falta.

Assim, nossa única tarefa é mostrar que o quadrado

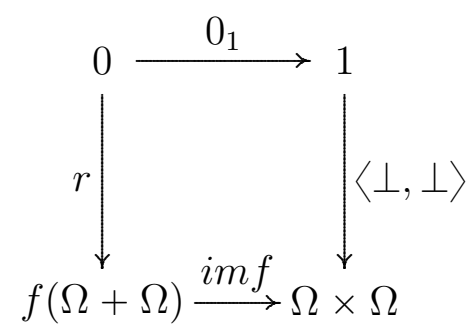

é um pullback.

Ocorre que, aparentemente, a prova desse fato necessita a introdução de hipóteses adicionais na nossa CTM $\mathcal{E}$. Essas hipóteses serão apresentadas na seção a seguir, definindo um tipo de CTM que denominaremos prototopos. O caso que resta para ser demonstrado será chamado de caso anômalo. 


\section{Prototopos}

A fim de lidar com o caso anômalo, vamos definir um tipo de categoria com morfismos verdade com algumas propriedades adicionais.

Dizemos que uma categoria $\mathcal{E}$ é um prototopos se e somente se $\mathcal{E}$ satisfaz os seguintes axiomas:

$(\mathrm{PT} 1) \mathcal{E}$ é uma categoria com morfismos verdade. Isto é, $\mathcal{E}$ é finitamente bicompleta, possui classificador de subobjetos e satisfaz o 0-Axioma.

(PT2) Em $\mathcal{E}$, pullbacks preservam epimorfismos. Isto é, se o quadrado

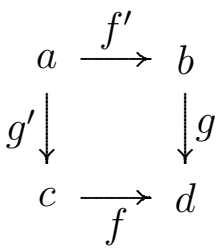

é um pullback e $f$ é um epimorfismo, então $f^{\prime}$ também é epimorfismo.

(PT3) Em $\mathcal{E}$, coprodutos preservam pullbacks. Isto é, se os diagramas abaixo

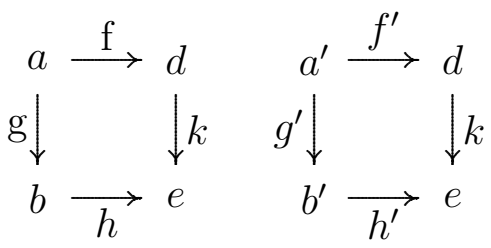

são pullbacks, então o quadrado

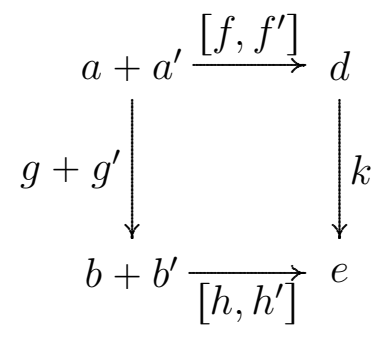

é um pullback também.

É preciso mencionar que (PT2) e (PT3) são propriedades que valem em topos e são consequências do Teorema Fundamental da Teoria de Topos: Se $\mathcal{E}$ é um topos e a é um $\mathcal{E}$-objeto, então a categoria slice $\mathcal{E} /$ a é também um topos.

Dessas propriedades, agora no ambiente de prototopos, segue um resultado que servirá de base paraa a demonstração do caso anômalo.

LEMA 15. Em qualquer prototopos $\mathcal{E}$, se o quadrado

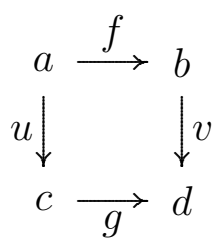

é um pullback, então existe um morfismo

$$
h: f(a) \rightarrow g(c)
$$


que torna o quadrado à direita do diagrama

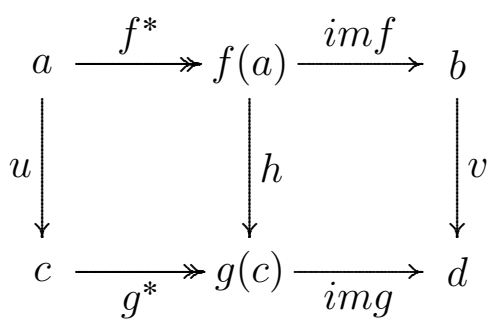

um pulback .

Prova. Considere o diagrama

$$
g(c) \stackrel{i m g}{\longmapsto} d \stackrel{v}{\longleftarrow} b .
$$

e construa um pullback do mesmo.

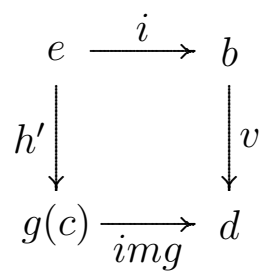

Considere, agora, o diagrama

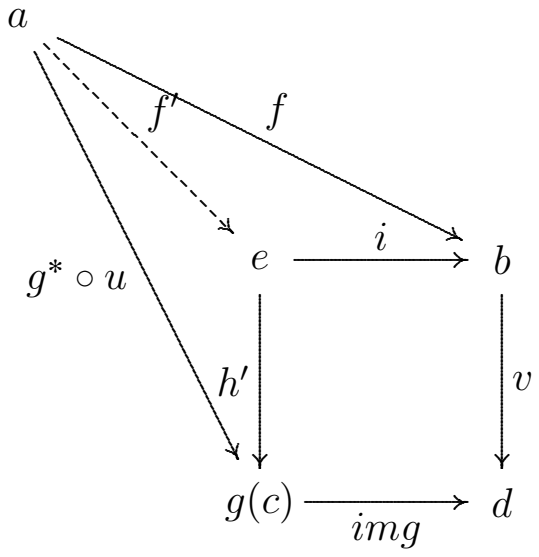

Como $i m g \circ g^{*}=g$ (fatoração epi-mono de $g$ ) e $g \circ u=f \circ v$ (hipótese), segue-se que $i m g \circ g^{*} \circ u=f \circ v$ (ou seja, o diagrama externo comuta). Como (A) é um pullback, então existe um único morfismo $f^{\prime}: a \rightarrow e$ tal que $i \circ f^{\prime}=f$. Temos, então, o seguinte diagrama comutativo:

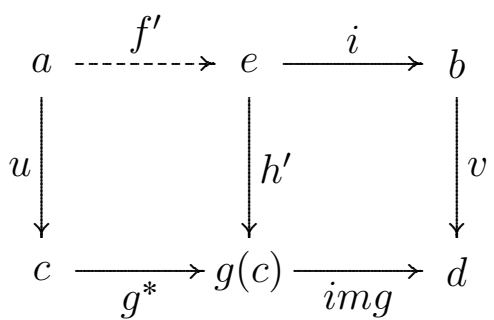

Como, por hipótese, o retângulo exterior é um pullback (pois, $i \circ f^{\prime}=f$ ) e o diagrama da direita também é um pullback (diagrama $(A)$ ), segue-se, pelo Lema do Pullback, que o quadrado da esquerda é um pullback.

Como $g^{*}$ é um epimorfismo, segue-se de (PT2) que $f^{\prime}$ é epimorfismo. Assim,

$$
f=i \circ f^{\prime}
$$

${ }^{7}$ Lembremos que $i m f \circ f^{*}$ e $i m g \circ g^{*}$ são decomposições epi-mono de $f$ e $g$, respectivamente; cuja existência está garantida pelo corolário 8. 
é uma fatoração epi-mono de $f$. ( $i$ é monomorfismo porque $i m g$ é mono e, em qualquer categoria, pullbacks preservam monomorfismos.)

Assim, pelo corolário 8, existe um único ismorfismo

$$
k: f(a) \cong e
$$

tal que o diagrama a seguir é comutativo:

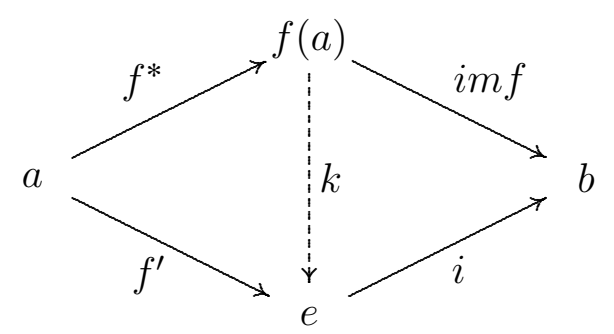

Definindo $h:=h^{\prime} \circ k$, temos o seguinte diagrama comutativo:

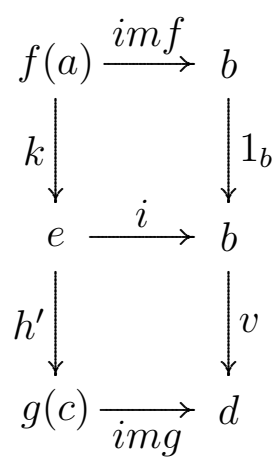

O quadrado inferior do diagrama acima é um pullback (diagrma (A)). Mostremos que o quadrado superior também é um pullback.

Considere, então, o quadrado

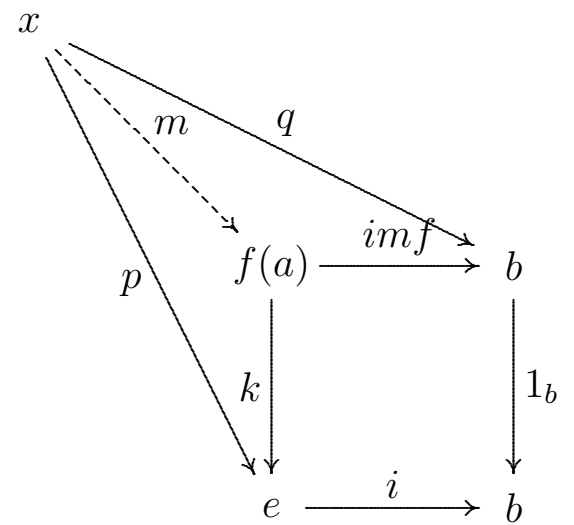

Já sabemos que $i \circ k=i m f=1_{b} \circ i m f$.

Sejam os morfismos $p: x \rightarrow e$ e $q: x \rightarrow b$ tais que $i \circ p=1_{b} \circ q=q$.

Seja $m:=k^{-1} \circ p$ (lembre que $k$ é iso).

Então, é claro que

$$
k \circ m=k \circ k^{-1} \circ p=1_{e} \circ p=p .
$$

Por outro lado,

$$
\begin{aligned}
i m f \circ m & =i m f \circ k^{-1} \circ p \\
& =i \circ k \circ k^{-1} \circ p \\
& =i \circ 1_{e} \circ p \\
& =i \circ p \\
& =q
\end{aligned}
$$


Assim, temos que $m$ fatora $p$ e $q$. Para ver que $m$ é único, suponha que exista $m^{\prime}: x \rightarrow f(a)$ tal que $k \circ m^{\prime}=p$. Então, $k \circ m^{\prime}=p=k \circ m$. Como $k$ é mono (pois é iso), segue-se que $m^{\prime}=m$.

Como os dois quadrados de $(B)$ são pullbacks, pelo Lema do Pullback, o retângulo é também um pullback e o lema está demonstrado.

\section{O caso anômalo}

ProposiçÃo 16. Em um prototopos $\mathcal{E}$, o quadrado dado pelo diagrama

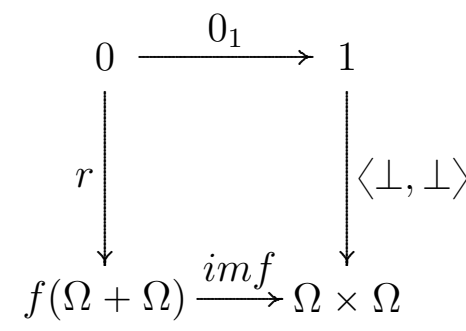

é um pullback.

Prova. Lembrando que $f$ é dado pelo coproduto

$$
f:=\left[\left\langle\top_{\Omega}, 1_{\Omega}\right\rangle,\left\langle 1_{\Omega}, \top_{\Omega}\right\rangle\right]: \Omega+\Omega \rightarrow \Omega \times \Omega
$$

e $i m f$ é o equalizador do pushout de $f$ por $f$

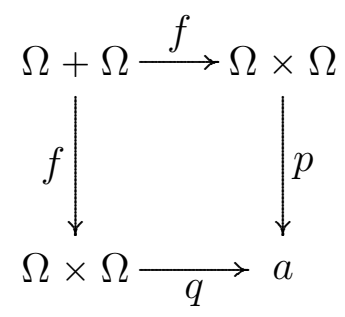

que é dado por

$$
f(\Omega+\Omega) \stackrel{i m f}{\longrightarrow} \Omega \times \Omega \stackrel{p, q \longrightarrow}{\longrightarrow} a .
$$

Considere, então, o seguinte diagrama

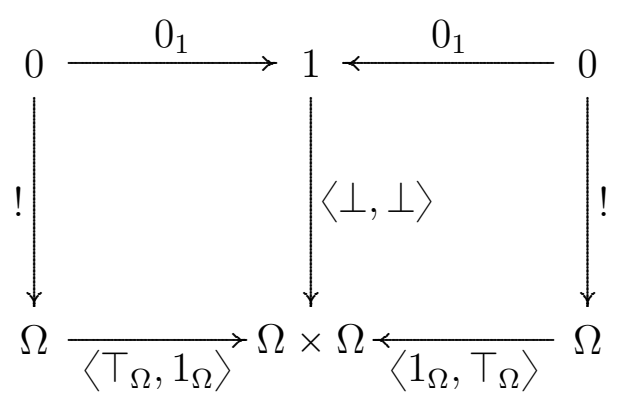


Vamos mostrar que ambos os quadrados são pullbacks. O argumento é análogo para ambos os casos. Então, consideremos o diagrama da esquerda.

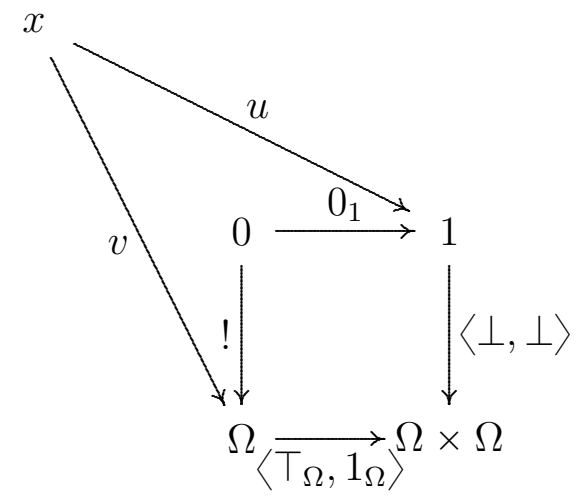

Considere os morfismos $v: x \rightarrow \Omega$ e $u: x \rightarrow 1$ tais que

$$
\left\langle\top_{\Omega}, 1_{\Omega}\right\rangle \circ v=\langle\perp, \perp\rangle \circ u \text {. }
$$

Tome o pullback que define $\perp$.

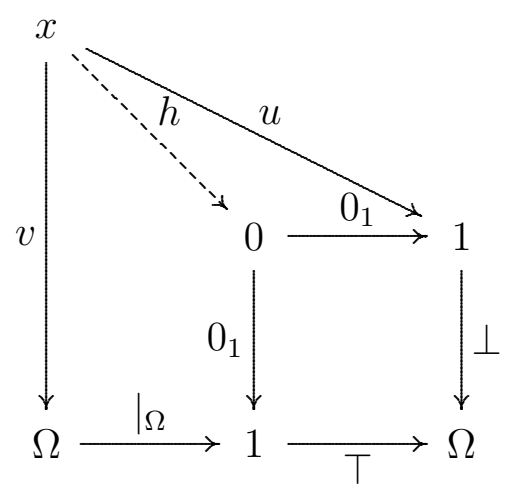

De (I) segue-se que:

$$
\left\langle\top_{\Omega} \circ v, 1_{\Omega} \circ v\right\rangle=\langle\perp \circ u, \perp \circ u\rangle,
$$

e isso nos fornece duas equações:

$$
\begin{array}{rlr}
\top_{\Omega} \circ v & =\perp \circ u & (I I a) \\
v & =\perp \circ u & (I I b)
\end{array}
$$

Pelo corolário 3, temos que $\perp \circ u=T_{\Omega} \circ v=\left.\top \circ\right|_{\Omega \circ v}$. Assim, como o quadrado de (B) é um pullback, existe um único $h: x \rightarrow 0$ tal que $0_{1} \circ h=\left.\right|_{\Omega} \circ v$ e $0_{1} \circ h=u$.

Pelo 0-Axioma, $x$ é inicial em $\mathcal{E}$ ( $h$ é iso e único).

Transportando $h$ para o diagrama $(\mathrm{A})$ e, como $x$ é inicial, $0_{1} \circ h=u$ (já sabíamos) e !० $h=v$. Logo, o quadrado de (A) é um pullback.

Com o mesmo argumento, temos que o quadrado à direita do diagrama (D) é um pullback também.

Usando, agora, o axioma (PT3) dos prototopos, segue-se que o seguinte diagrama é um pullback

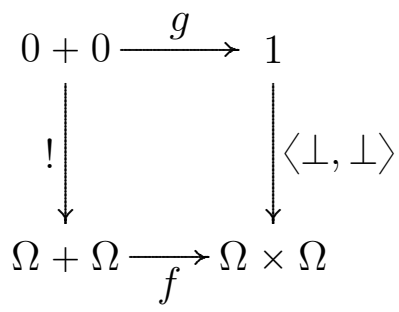

em que $g=[0,0]$ e $f=\left[\left\langle\top_{\Omega}, 1_{\Omega}\right\rangle,\left\langle 1_{\Omega}, \top_{\Omega}\right\rangle\right]$. 
Aplicando o lema 15, segue-se que existe $r: g(0+0) \rightarrow f(\Omega+\Omega)$ que torna o quadrado da direita do diagrama

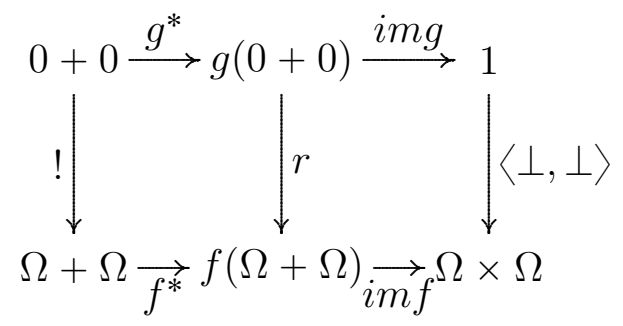

um pullback.

Mas, $0+0 \cong 0$ e $g(0+0) \cong g(o) \cong 0$. Assim, temos que o quadrado

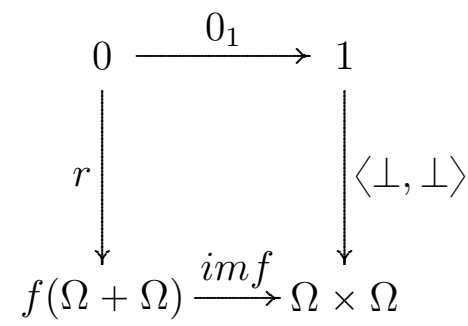

é um pullback. Como queríamos demonstrar.

O raciocínio acima resolve o caso anômalo estabelecendo que $f_{\vee} \circ\langle\perp, \perp\rangle=\perp$. Assim, em prototopos, o morfismo disjunção comporta-se, com respeito aos valores de verdade $T$ e $\perp$, de acordo com a tabela

\begin{tabular}{cc|c}
$x$ & $y$ & $f_{\vee} \circ\langle x, y\rangle$ \\
\hline$\top$ & $\top$ & $\top$ \\
$\top$ & $\perp$ & $\top$ \\
$\perp$ & $\top$ & $\top$ \\
$\perp$ & $\perp$ & $\perp$
\end{tabular}

que corresponde à tabela usual da disjunção na lógica proposicional clássica.

Segue-se, então, uma conclusão geral: Em categorias com morfismos verdade, podem ser definidos todos os morfismos que correspondem às funções de verdade da negação, conjunção, implicação material e disjunção das linguagens proposicionais, fornecendo assim uma semântica categorial para as mesmas. Além disso, em CTM's, os morfismos verdade comportam-se com relação aos valores de verdade exatamente como se comportam as funções clássicas, com uma única exceção correspondente ao que denominamos de caso anômalo. Para este, introduzimos a noção de prototopos que possui a propriedade desejada.

Resta investigar qual é a lógica subjacente à semântica de prototopos. É um resultado conhecido que no caso de topos, a lógica subjacente é o cálculo proposicional intuicionista ${ }^{8}$. Como todo topos é também um prototopos, segue-se que na semântica de prototopos o princípio do terceiro excluído também nao vale. Mas, ainda não está totalmente claro que a lógica proposicional intuicionista caracteriza essa semântica. Isso será investigado em trabalhos futuros.

Para completar o presente trabalho vamos mostrar que em CTM's vale um resultado análogo ao teorema fundamental da teoria de topos.

\footnotetext{
${ }^{8}$ Ver Boileau \& Joyal [3].
} 


\section{O teorema fundamental para CTM}

Considere uma categoria $\mathcal{C}$ qualquer e $X$ um $\mathcal{C}$-objeto. Definimos a categoria slice de $\mathcal{C}$ por $X$, denotada por $\mathcal{C} / X$, de forma que:

- $\mathcal{C} / X$-objetos: são os $\mathcal{C}$-morfismos da forma $A \stackrel{a}{\rightarrow} X$;

- $\mathcal{C} / X$-morfismos: dados os $\mathcal{C} / X$-objetos $A \stackrel{a}{\rightarrow} X$ e $B \stackrel{b}{\rightarrow} X$, um $\mathcal{C} / X$-morfismo $f: a \rightarrow b$ é simplesmente um $\mathcal{C}$-morfismo $f: A \rightarrow B$ tal que o seguinte diagrama comuta

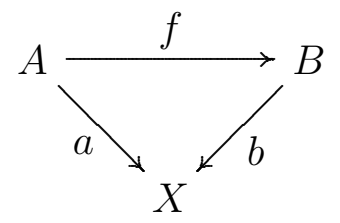

isto é, $b \circ f=a$.

Composição de morfismos em $\mathcal{C} / X$ é a composição usual de morfismos em $\mathcal{C}$ e, também, identidades em $\mathcal{C} / X$ são identidades em $\mathcal{C}$.

Vamos demonstrar, para as CTM's, um teorema análogo ao teorema fundamental da teoria de topos ${ }^{9}$.

TheOrem 17. Se E é uma categoria com morfismos verdade e X é um E-objeto, então a categoria slice $\mathcal{E} / X$ é também uma categoria com morfismos verdade.

Prova. É preciso fazer várias verificações. Lembremos primeiramente que uma condição necessária e suficiente para uma categoria seja finitamente completa é que ela possua objetos terminais, produtos de pares de objetos e equalizadores de pares de morfismos. $\mathrm{O}$ mesmo vale para o caso dual, da cocompletude finita.

(I) O morfismo indentidade $1_{X}: X \rightarrow X$ é terminal em $\mathcal{E} / X$. De fato, dado um $\mathcal{E} / X$ objeto $A \stackrel{a}{\rightarrow} X$, esse morfismo é o único $\mathcal{E} / X$-morfismo que faz o diagrama abaixo comutar.

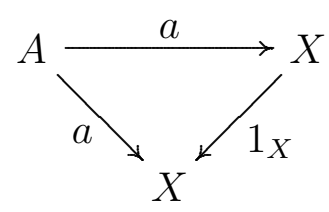

Assim, $a$ é o único morfismo de $a$ em $1_{X}$, isto é, $1_{X}$ é terminal em $\mathcal{E} / X$.

(II) Considere dois $\mathcal{E} / X$-objetos $A \stackrel{a}{\rightarrow} X$ e $B \stackrel{b}{\rightarrow} X$. Como $\mathcal{E}$ é finitamente completa, construa o pullback

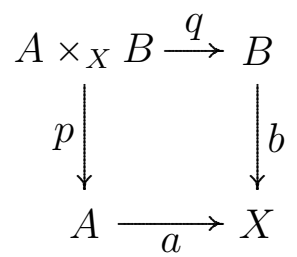

Afirmação: $O$ E-objeto $a \circ p: A \times_{X} B \rightarrow X$ junto com os $\mathcal{E}$-morfismos $p: A \times_{X} B \rightarrow A e$ $q: A \times_{X} B \rightarrow B$ formam um produto de a e $b$ em $\mathcal{E} / X$.

\footnotetext{
${ }^{9}$ Ver Freyd [5] e Kock \& Wraith [8].
} 
De fato, para ver que temos um produto, considere o diagrama

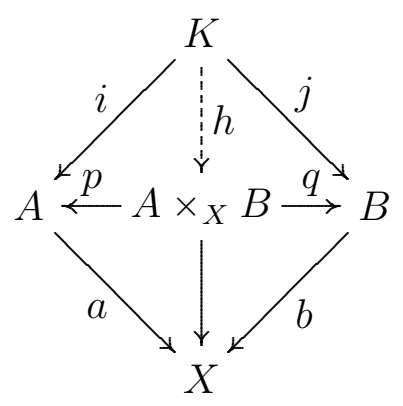

e suponha que $a \circ i=b \circ j$ (condição para $i$ e $j$ serem morfismos em $\mathcal{E} / X$ ).

Transportando para o diagrama do pullback em $\mathcal{E}$ (ver diagrama)

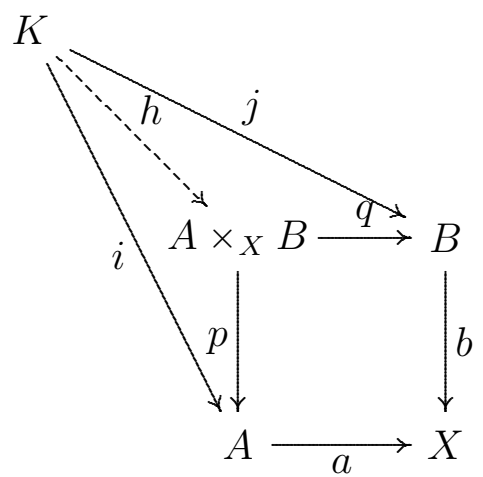

temos que existe um único morfismo $h: K \rightarrow A \times_{X} B$ tal que $p \circ h=i$ e $q \circ h=j$. E essa é a condição necessária para a existência de produto em $\mathcal{E} / X$. Assim, um produto em $\mathcal{E} / X$ é apenas um pullback em $\mathcal{E}$.

(III) Considere dois $\mathcal{E} / X$-objetos $A \stackrel{a}{\rightarrow} X$ e $B \stackrel{b}{\rightarrow} X$ e dois $\mathcal{E} / X$-morfismos paralelos $f, g: A \rightarrow B$ de forma que o seguinte diagrama comuta:

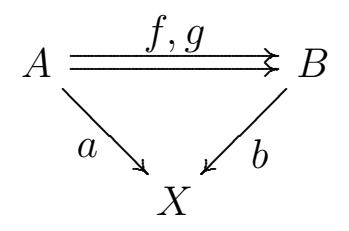

isto é, $b \circ f=a=b \circ g$.

Como $\mathcal{E}$ é finitamente completa, tome um equalizador de $f$ e $g$ em $\mathcal{E}$.

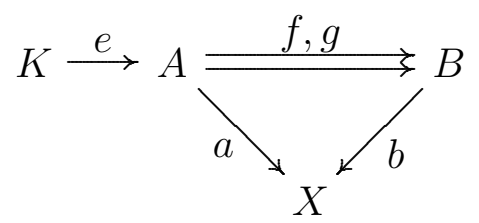

Afirmação: $O \mathcal{E} / X$-morfismo $e: K \rightarrow A$ cujo dominio é o $\mathcal{E} / X$-objeto a $\circ e: K \rightarrow X$ é um equalizador de $f$ e $g$ em $\mathcal{E} / X$.

A prova segue diretamente do fato de que e é um equalizador de $f$ e $g$ em $\mathcal{E}$. Assim, equalizadores em $\mathcal{E} / X$ nada mais são do que equalizadores em $\mathcal{E}$.

Segue-se de (I), (II) e (III) que: $\mathcal{E} / X$ é uma categoria finitaente completa.

Para a cocompletude finita de $\mathcal{E} / X$, note primeiramente que se 0 é inicial em $\mathcal{E}$, o único morfismo $0 \rightarrow X$ é inicial em $\mathcal{E} / X$. 
De fato, dado um $\mathcal{E} / X$-morfismo $A \stackrel{a}{\rightarrow} X$, o morfismo $0 \stackrel{0_{A}}{\rightarrow} A$ é o único morfismo que faz o diagrama

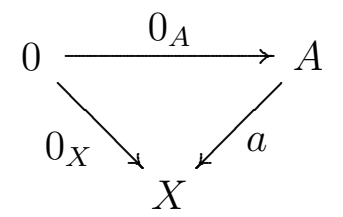

comutar $a \circ 0_{A}=0_{X}$, pois 0 é inicial em $\mathcal{E}$. Assim, objetos iniciais em $\mathcal{E} / X$ são $\mathcal{E}$-morfismos cujo domínio é inicial em $\mathcal{E}$.

Assim, temos:

(IV) $\mathcal{E} / X$ tem objetos iniciais.

Para coprodutos e coequalizadores temos as construções anáogas aos seus duais, de modo que:

(V) Um coproduto em $\mathcal{E} / X$ é um pushout em $\mathcal{E}$.

(VI) Um coequalizador em $\mathcal{E} / X$ é um coequalizador m $\mathcal{E}$.

Assim, temos também que: $\mathcal{E} / X$ é uma categoria finitamente cocompleta.

(VII) Para ver que $\mathcal{E} / X$ tem classificador de subobjetos, note primeiramente que $1_{X}$ é um objeto terminal em $\mathcal{E} / X$. Como a identidade $1_{X}$ é um monomorfismo, aplicando o $\Omega$-Axioma, que vale em $\mathcal{E}$, temos o pullback dado pelo diagrama:

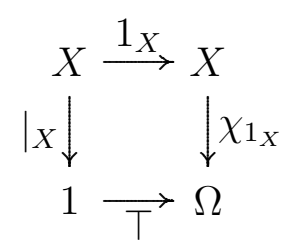

de forma que $\chi_{1_{X}}=\top_{X}:=\left.\top \circ\right|_{X}: X \rightarrow \Omega$.

Defina o $\mathcal{E}$-morfismo $T / X:=\left\langle\top_{X}, 1_{X}\right\rangle: X \rightarrow \Omega \times X$. dado pelo diagrama produto:

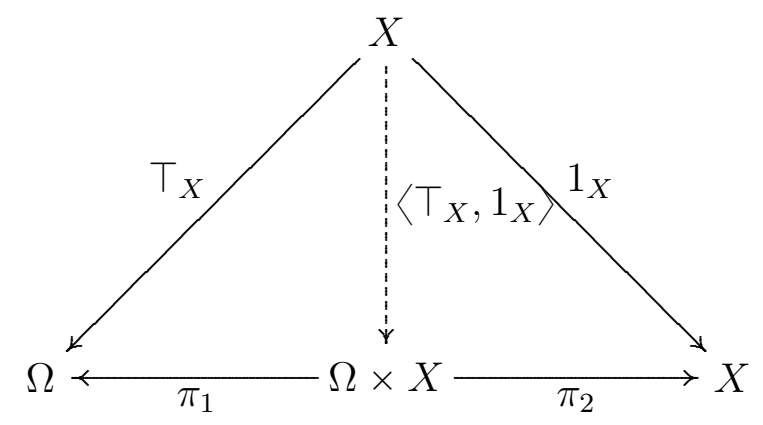

Seja $\Omega_{0}=\pi_{2}: \Omega \times X \rightarrow X$, a segunda projeção do produto.

Dados dois $\mathcal{E} / X$-objetos $A \stackrel{a}{\rightarrow} X$ e $B \stackrel{b}{\rightarrow} X$, um monomorfismo $\gamma: a \longmapsto b$ em $\mathcal{E} / X$ é simplesmente um monomorfismo $\gamma: A \longmapsto B$ em $\mathcal{E}$ tal que o seguinte diagrama comuta:

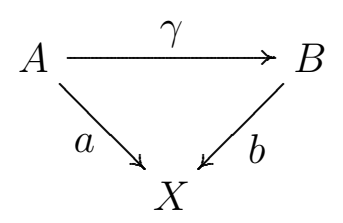

Assim, $\gamma$ é um subobjeto de $B$ em $\mathcal{E}$. 
Aplicando novamente o $\Omega$-Axioma em $\gamma$ temos o seguinte pullback em $\mathcal{E}$ :

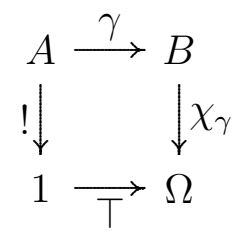

Para transpor a construção para $\mathcal{E} / X$, para cada monomorfismo $\gamma: A \longmapsto B$ em $\mathcal{E}$ defina o $\mathcal{E} / X$-morfismo $\gamma^{*}=\left\langle\chi_{\gamma}, b\right\rangle: B \rightarrow \Omega \times X$ dado pelo seguinte produto:

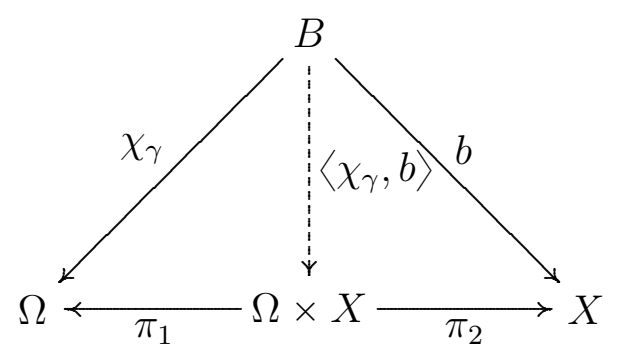

Assim, $\gamma^{*}=\left\langle\chi_{\gamma}, b\right\rangle$ é um morfismo em $\mathcal{E} / X$ da forma $\gamma^{*}: b \rightarrow \Omega_{0}\left(=\pi_{2}\right)$.

Mas, então, o diagrama

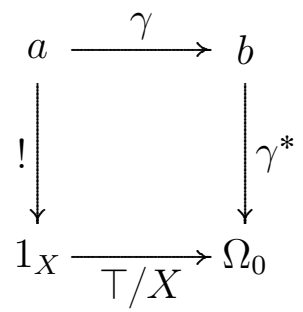

é um pullback em $\mathcal{E} / X$ e a propriedade universal provém da universalidade do $\Omega$-Axioma em $\mathcal{E}$.

Assim, $T / X: 1_{X} \rightarrow \Omega_{0}$ é um classificador de subobjetos para $\mathcal{E} / X$. Segue-se que: $\mathcal{E} / X$ tem classificador de subobjetos.

(VIII) Resta verificar o 0-Axioma.

Lembremos que em $\mathcal{E} / X$, um objeto inicial é um morfismo $0 \stackrel{0_{X}}{\rightarrow} X$, em que 0 é inicial em $\mathcal{E}$.

Considere um $\mathcal{E} / X$-morfismo $a \stackrel{f}{\rightarrow} 0_{X}$ cujo domínio é $A \stackrel{a}{\rightarrow} X$. Isto significa que o diagrama

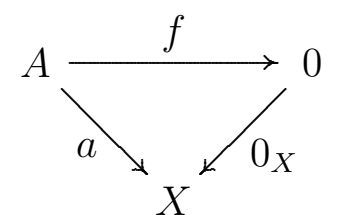

comuta. Como o 0-Axioma vale para $\mathcal{E}, f$ é iso. Logo, $f$ é iso em $\mathcal{E} / X$. Assim, temos que: $O$ 0-Axioma vale para $\mathcal{E} / X$.

Assim, temos que $\mathcal{E} / X$ é uma categoria com morfismos verdade.

Para finalizar, observemos que, em teoria de topos, os axiomas (PT2) e (PT3) dos prototopos são consequências do teorema fundamental para esta teoria. Fica aberta a questão se esses axiomas são também válidos em categorias com morfismos verdade em virtude do teorema acima. Se esse for o caso as noções de prototopos e categorias com morfismos verdade seriam equivalentes. 


\section{Referências Bibliográficas}

[1] Awodey, S. (2010). Category theory. Oxford University Press.

[2] Bell, J. L. (2008). Toposes and local set theories: an introduction. Courier Corporation.

[3] Boileau, A. e Joyal, A. (1981). La logique des topos. The Journal of Symbolic Logic, vol 46(1), pp 6-16.

[4] de Souza, E. G. (2018) Sobre a noção categorial de prototopos. Revista de Filosofia Moderna e Contemporânea. Vol 6. PP 105-114.

[5] Freyd, P. (1972). Aspects of topoi. Bulletin of the Australian Mathematical Society, vol 7, pp 1-76.

[6] Goldblatt, R. (2006). Topoi: the categorical analysis of logic. Dover Publications, Inc.

[7] Herrlich, H. e Strecker, G. E. (1973). Category theory. Allyn and Bacon Inc.

[8] Kock, A. e Wraith, G. C. (1971). Elementary toposes. Aarhaus Lecture Note Series, 30.

[9] MacLane, S. (1971). Categories for the working mathematician. Springer-Verlag.

[10] MacLane, S. e Moerdijk, I. (2012). Sheaves in geometry and logic: A first introduction to topos theory. Springer-Verlag.

[11] MacLarty, C. (1995). Elementary categories, elementary toposes. Clarendon Press. 